\title{
A Correction Method for Unsteady Transonic Aerodynamics
}

\author{
Upender K. Kaul* and Nhan T. Nguyen** \\ NASA Ames Research Center, USA.
}

This paper presents a new method for transonic pitching airfoils based on a RANS CFD study and the Theodorsen model of an oscillating pitching flat plate. This study quantifies the deviation of the lift coefficient predictions using CFD from that obtained using the Theodorsen model, which is based on the incompressible potential flow assumption. The present method corrects this theoretical model by modulating the Theodorsen functions by coefficient functions that depend on the reduced frequency and the Mach number. It is demonstrated that the modified theoretical model predicts lift coefficient in good agreement with the CFD results in the Mach number range from incompressible $(M=0.2)$ to transonic $(M=0.755)$ flow for a range of reduced frequencies typical of transonic flutter. The simulations are first validated by comparing pitching NACA0012 airfoil results with experimental results at transonic flight conditions, which establishes the requirements for a grid converged unsteady transonic solution. The hysteresis loop, $C_{l}$ versus $\alpha$, attains a grid independent solution that compares well with experiment. The present correction method will guide the development of a new state space model for the Variable Camber Continuous Trailing Edge Flap (VCCTEF) system and eventually a new transfer function that will be incorporated in a new aeroelastic framework leading to an appropriate transonic flutter model for use in the future aircraft systems in development under the NASA Advanced Air Transportation Technologies (AATT) project.

\section{Introduction}

Transonic flutter is a topic of high interest for aircraft design. Doublet lattice methods are frequently used in flutter analysis that can accurately predict low-speed flutter where the flow is entirely subsonic at low Mach number [1]. Transonic flutter, on the other hand, is a pacing item in transport aircraft design. Many methods for transonic flutter have been developed ranging from doublet-lattice methods with steady state transonic correction to high-fidelity CFD coupled to structural dynamic models of wing structures. ${ }^{2-4}$ While the transonic flutter prediction can be more accurate with high-fidelity CFD methods ${ }^{5,6}$ than with unsteady potential flow methods, the computational cost is high. In many applications that involve design optimization with flutter constraints, the computational cost associated with high-fidelity CFD presents a barrier. Therefore, computationally efficient methods for transonic flutter prediction continue to be of high interest to the aircraft design community. In this work, we are proposing a method for extending the classical Theodorsen's theory of unsteady aerodynamics for incompressible flow [7] to transonic flow. The method proposes a modified Theodorsen's function to correct for changes in the amplitude and phase shift of the circulatory lift due to transonic flow. The method derives, partly, from a reduced set of steady state CFD simulations that are made a priori, as an input to the new method. Unsteady CFD simulations are performed using OVERFLOW ${ }^{8-10}$ for selected reduced frequencies and Mach numbers for the NACA0012 airfoil for validation of the proposed method. The proposed method demonstrates good agreement with the CFD simulation results. Using this method, computationally efficient methods for transonic flutter prediction can be developed by incorporating appropriate transonic corrections to the Theodorsen's function using a strip-theory approach.

\footnotetext{
* Computational Aerosciences Branch, NASA Advanced Supercomputing (NAS) Division; Associate Fellow, AIAA

** Intelligent Systems Division; Associate Fellow, AIAA
} 


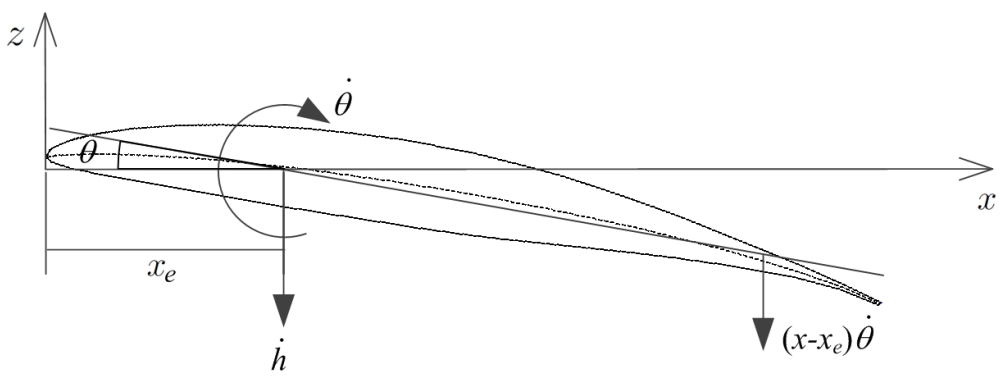

Figure 1: Downwash of Oscillating Airfoil

\section{Method and Formulation}

The starting point for the correction method proposed in this paper is the Theodorsen model, which requires that the Kutta condition be enforced at the sharp trailing edge, that the wake is flat and that the wake vortices convect downstream at $V_{\infty}$. In our study of the VCCTEF, the trailing edge is blunt and, therefore, there seems to be an inherent limitation in the application of the Theodorsen model. However, as we have shown in this paper, this model is useful for a variety of airfoils, such as NACA series airfoils with blunt trailing edge. If the flow separates tangentially from the top and bottom edges of the trailing edge face, the Kutta condition is practically obeyed. Any discussion of the Kutta condition in this paper should be understood with this aspect in mind.

The zero-normal velocity condition on the airfoil is satisfied by source/sink singularities on the airfoil. The pitching motion of the airfoil determines the strength of the source/sink distribution. This is similar to the mixing layer flow over a flat plate subject to sinuous oscillations imposed at the trailing edge, where the zero normal velocity on the flat plate is enforced by a vortex sheet whose density changes with time ${ }^{11}$. The pressure difference caused by the associated potential gives rise to the "non-circulatory lift", which excludes the effect of the wake on the lift on the airfoil. This non-circulatory lift is a result of the so-called apparent mass effect due to inertia associated with the airfoil motion. It reflects the pressure forces required to accelerate the fluid near the airfoil. This lift dominates for the case when the normal velocity due to the pitching motion is a strong function of time, for example, for higher values of reduced frequency. The vortex singularity distribution in the wake gives rise to the lift which together with the quasi-steady lift forms the "circulatory lift". The quasi-steady lift is generated if the normal velocity due to the pitching motion is almost time-independent, i.e., $\partial v / \partial t \approx 0$, and the wake does not contribute to the lift. So, for lower values of reduced frequency, the circulatory lift becomes dominant.

For oscillatory airfoil, the singularity distributions on the airfoil and in the wake change with time, but satisfy the two constraints (zero normal velocity on the airfoil and Kutta condition) for all time. For flutter problems, the angle of attack is assumed to be small enough so that the flow separates approximately tangentially from the trailing edge.

When an airfoil undergoes oscillations, the circulatory lift is influenced by the frequency of oscillation. Consider the unsteady motion of an oscillatory pitching and plunging airfoil with an angular velocity $\dot{\theta}$ and plunging velocity $\dot{h}$ where $\theta$ is the instantaneous pitch angle, positive nose-up, and $h$ is the vertical displacement of the airfoil, positive downward. This is shown in Fig.

The slope of the camber line is related to the downwash as

$$
\frac{d z}{d x}=-\frac{V_{z}}{V_{\infty}}=-\left[\theta+\frac{\left(x-x_{e}\right) \dot{\theta}}{V_{\infty}}+\frac{\dot{h}}{V_{\infty}}\right]
$$

Based on thin-airfoil aerodynamic theory for incompressible flow, the circulatory lift coefficient due to the effective camber change is evaluated by

$$
C_{l}=-\frac{C(k) c_{l_{\alpha}}}{\pi} \int_{0}^{\pi} \frac{d z}{d x}(1-\cos \varphi) d \varphi=\frac{C(k) C_{l_{\alpha}}}{\pi} \int_{0}^{\pi}\left[\theta+\frac{\left(x-x_{e}\right) \dot{\theta}}{V_{\infty}}+\frac{\dot{h}}{V_{\infty}}\right](1-\cos \varphi) d \varphi
$$



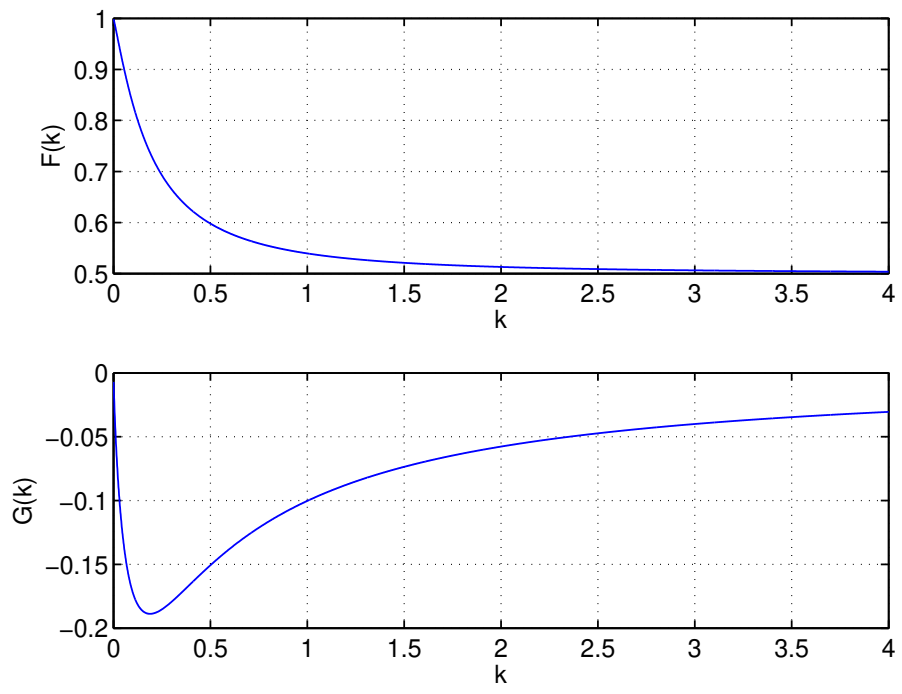

Figure 2: Theodorsen's Function

This equation is valid for small-amplitude oscillations for which the Kutta condition at the trailing edge is generally assumed to be valid. For incompressible flow, $C_{l_{\alpha}}=2 \pi$. The function $C(k)$ is the complex-valued Theodorsen's function [7], the exact expression of which is given by ${ }^{7}$

$$
C(k)=\frac{H_{1}^{(2)}(k)}{H_{1}^{(2)}(k)+i H_{0}^{(2)}(k)}=F(k)+i G(k)
$$

where $H_{0}^{(2)}(k)$ and $H_{1}^{(2)}(k)$ are the Hankel functions, and $k$ is called reduced frequency which is defined as

$$
k=\frac{\omega c}{2 V_{\infty}}
$$

The functions $F(k)$ and $G(k)$ are plotted in Fig. 2. It is noted that $F(0)=1$ and $G(k)=0$ for steady state aerodynamics corresponding to $k=0$. As $k \rightarrow \infty, F(k) \rightarrow \frac{1}{2}$ and $G(k) \rightarrow 0$. So the magnitude of the unsteady circulatory lift coefficient is always less than the magnitude of the steady state lift coefficient.

Let $x-x_{e}=\frac{c}{2}\left(\cos \varphi_{e}-\cos \varphi\right)$. Then, this integral yields

$$
C_{l}=C(k) C_{l_{\alpha}}\left[\theta+\left(\frac{3 c}{4}-x_{e}\right) \frac{\dot{\theta}}{V_{\infty}}+\frac{\dot{h}}{V_{\infty}}\right]
$$

Thus, the effective angle of attack of a pitching and plunging airfoil is equal to

$$
\alpha_{e}=\theta+\left(\frac{3 c}{4}-x_{e}\right) \frac{\dot{\theta}}{V_{\infty}}+\frac{\dot{h}}{V_{\infty}}
$$

The three-quarter-chord point is the location where the effective downwash of an oscillating airfoil acts. The circulatory lift force acting on the airfoil in incompressible flow is then given by

$$
L_{c i r c}=C_{l} q_{\infty} c=C(k) C_{l_{\alpha}} q_{\infty} c\left[\theta+\left(\frac{3 c}{4}-x_{e}\right) \frac{\dot{\theta}}{V_{\infty}}+\frac{\dot{h}}{V_{\infty}}\right]
$$

As the airfoil undergoes pitching and plunging motion, an opposing inertial force is created due to the 


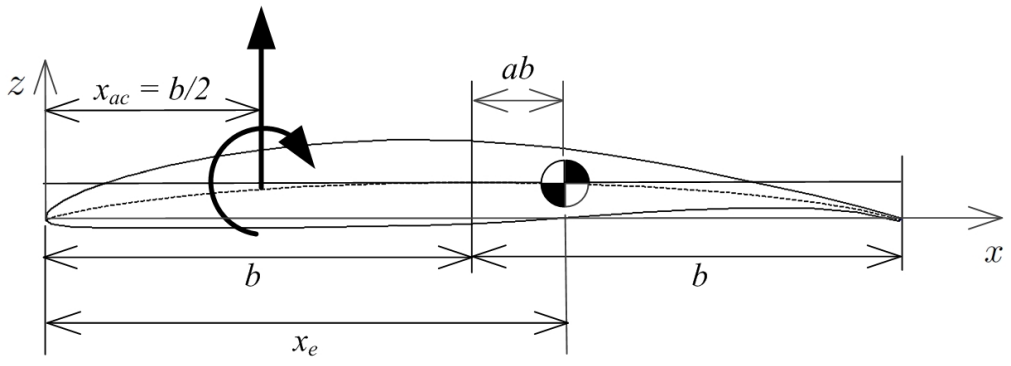

Figure 3: Theodorsen's Airfoil Convention

surrounding air acting on the airfoil. This unsteady lift force is called non-circulatory due to the apparent mass effect.

For the non-circulatory lift, we express the local angle of attack along the airfoil chord as [12]

$$
\alpha_{c}(x, t)=\frac{V_{z}}{V_{\infty}}=\alpha_{1}(t)+\alpha_{2}(x, t)
$$

where $\alpha_{1}=\theta+\frac{\dot{h}}{V_{\infty}}$ and $\alpha_{2}=\frac{\left(x-x_{e}\right) \dot{\theta}}{V_{\infty}}$.

According to Bisplinghoff et al. [13], we define the velocity potential on the upper surface as [12]

$$
\phi(x, t)=\frac{V_{\infty} c}{2}\left[\alpha_{1}(t)+\frac{\alpha_{2}(x, t)+\alpha_{2}\left(x_{m}, t\right)}{2}\right] \sqrt{1-\xi^{2}}
$$

where $\xi=\frac{2\left(x-x_{m}\right)}{c}$ and $x_{m}=\frac{c}{2}$.

The non-circulatory lift is evaluated as

$$
\begin{aligned}
L_{\text {noncirc }}=\rho_{\infty} c \frac{\partial}{\partial t} \int_{-1}^{1} \phi d \xi=\frac{\rho_{\infty} V_{\infty} c^{2}}{2} \frac{\partial}{\partial t} & \int_{-1}^{1}\left[\alpha_{1}+\frac{1}{2} \alpha_{2}+\frac{1}{2} \alpha_{2}\left(x_{m}, t\right)\right] \sqrt{1-\xi^{2}} d \xi \\
& =\frac{\rho_{\infty} V_{\infty} \pi c^{2}}{4} \dot{\alpha}_{c}\left(x_{m}, t\right)=\rho_{\infty} \frac{\pi c^{2}}{4}\left[V_{\infty} \dot{\theta}+\left(x_{m}-x_{e}\right) \ddot{\theta}+\ddot{h}\right]
\end{aligned}
$$

The non-circulatory lift in Eq. 11 arises out of considering a volume of air cylinder with a radius $b$ that surrounds the airfoil. Then the non-circulatory lift force is equal to the apparent mass of the air volume times the acceleration of the airfoil acting at the mid-chord point due to the pitching and plunging motion.

Let $e=x_{e}-\frac{c}{4}, e_{m}=\frac{c}{2}-x_{e}$, and $e_{c}=\frac{3 c}{4}-x_{e}$. Then, the total lift force is expressed as ${ }^{12}$

$$
L=L_{\text {circ }}+L_{n o n c i r c}=C(k) C_{l_{\alpha}} q_{\infty} c\left(\theta+e_{c} \frac{\dot{\theta}}{V_{\infty}}+\frac{\dot{h}}{V_{\infty}}\right)+\rho_{\infty} \frac{\pi c^{2}}{4}\left(V_{\infty} \dot{\theta}+e_{m} \ddot{\theta}+\ddot{h}\right)
$$

In aeroelasticity, Theodorsen's airfoil convention is frequently used. The airfoil chord is $c=2 b$ and the location of the elastic center is at $x_{e}=(1+a) b$ from the leading edge, as shown in Fig. 3. The parameter $a$ is positive if the elastic center is behind the mid-chord point and is negative if the elastic center is forward of the mid-chord point. The aerodynamic center for incompressible flow is at the quarter-chord point $x_{a c}=\frac{b}{2}$. Thus, the total lift force according to Theodorsen is expressed equivalently as

$$
L=2 C(k) C_{l_{\alpha}} q_{\infty} b\left[\theta+\left(\frac{1}{2}-a\right) \frac{b \dot{\theta}}{V_{\infty}}+\frac{\dot{h}}{V_{\infty}}\right]+\rho_{\infty} \pi b^{2}\left(V_{\infty} \dot{\theta}-a b \ddot{\theta}+\ddot{h}\right)
$$

Let $\tau=\frac{t V_{\infty}}{c}$ be a normalized time variable. We now consider an oscillating airfoil in transonic flow with a harmonic angle of attack 


$$
\alpha=\bar{\alpha}+\alpha_{0} \sin \omega t=\bar{\alpha}+\alpha_{0} \sin 2 k \tau
$$

where $\bar{\alpha}$ is the mean angle of attack.

For transonic flow, it is hypothesized that the circulatory lift resembles that for incompressible flow but with a different amplitude and phase shift. To capture the changes in the amplitude and phase shift, we propose a modified Theodorsen's function as

$$
C_{T}\left(k, M, \frac{t}{c}\right)=C_{F}\left(k, M, \frac{t}{c}\right) F(k)+i C_{G}\left(k, M, \frac{t}{c}\right) G(k)
$$

where $C_{T}\left(k, M, \frac{t}{c}\right)$ is a modified Theodoren's function for transonic flow. The functions $C_{F}\left(k, M, \frac{t}{c}\right)$ and $C_{G}\left(k, M, \frac{t}{c}\right)$ represent the amplitude and phase shift correction factors, respectively, and are assumed to be dependent on the type of airfoil, reduced frequency, Mach number, and airfoil thickness.

The circulatory lift in transonic flow is then modified according to

$$
\begin{aligned}
C_{l_{c i r c}} & =\bar{C}_{l}+C_{l_{\alpha}}\left[C_{F} F(k)\left(\alpha-\bar{\alpha}+\frac{e_{c}}{c} \frac{d \alpha}{d \tau}\right)+\frac{C_{G} G(k)}{2 k}\left(\frac{d \alpha}{d \tau}+\frac{e_{c}}{c} \frac{d^{2} \alpha}{d \tau^{2}}\right)\right] \\
& =C_{l_{0}}+C_{l_{\alpha}} \bar{\alpha}+C_{l_{\alpha}} \alpha_{0}\left[C_{F} F(k)\left(\sin 2 k \tau+\frac{e_{c}}{c} 2 k \cos 2 k \tau\right)+C_{G} G(k)\left(\cos 2 k \tau-\frac{e_{c}}{c} 2 k \sin 2 k \tau\right)\right]
\end{aligned}
$$

The quantity $\bar{C}_{l}$ is the mean lift coefficient due to the airfoil camber and the mean angle of attack $\bar{\alpha}$.

We assume that the inertial force acting on the airfoil is the same in transonic flow as in incompressible flow and is given by

$$
C_{l_{\text {noncirc }}}=\frac{\pi}{2}\left(\frac{d \alpha}{d \tau}+\frac{e_{m}}{c} \frac{d^{2} \alpha}{d \tau^{2}}\right)=\pi k \Delta \alpha\left(\cos 2 k \tau-\frac{e_{m}}{c} 2 k \sin 2 k \tau\right)
$$

The amplitude and phase shift of the oscillating circulatory lift are computed as

$$
\begin{aligned}
&\left|C_{l_{c i r c}}-\bar{C}_{l}\right|=\left|\Delta C_{l_{c i r c}}\right|= C_{l_{\alpha}} \alpha_{0} \sqrt{\left[C_{F} F(k)-C_{G} G(k) \frac{e_{c}}{c} 2 k\right]^{2}+\left[C_{G} G(k)+C_{F} F(k) \frac{e_{c}}{c} 2 k\right]^{2}} \\
& \measuredangle\left(\Delta C_{l_{c i r c}}\right)=\tan ^{-1} \frac{C_{G} G(k)+C_{F} F(k) \frac{e_{c}}{c} 2 k}{C_{F} F(k)-C_{G} G(k) \frac{e_{c}}{c} 2 k}
\end{aligned}
$$

The phase shift is also given by

$$
\measuredangle\left(\Delta C_{l_{\text {circ }}}\right)=2 k \tau_{\text {lag }}
$$

where $\tau_{\text {lag }}$ is the lag time between the oscillating circulatory lift and the quasi-steady state lift which is expressed as

$$
\Delta \bar{C}_{l}=C_{l_{\alpha}} \alpha_{0} \sin 2 k \tau
$$

When the quasi-steady state lift reaches its maximum amplitude at some normalized time $\tau=\tau_{0}$ after the flow establishes a steady state, the oscillating circulatory lift reaches its maximum ampltitude later at $\tau=\tau_{0}-\tau_{\text {lag }}$. Thus, $\tau_{\text {lag }}<0$ when the oscillating circulatory lift lags the quasi-steady state lift.

These two nonlinear equations can be solved for $C_{F}$ and $C_{G}$ if the amplitude and phase shift of the circulatory lift are given. CFD simulations are performed to compute the amplitude and phase shift of the NACA0012 airfoil. The solutions are given by

$$
\begin{aligned}
C_{F} & =\frac{\left|\Delta C_{l_{c i r c}}\right|\left(\cos \measuredangle\left(\Delta C_{l_{c i r c}}\right)+\frac{e_{c}}{c} 2 k \sin \measuredangle\left(\Delta C_{l_{c i r c}}\right)\right)}{c_{l_{\alpha}} \Delta \alpha F(k)\left[1+\left(\frac{e_{c}}{c} 2 k\right)^{2}\right]} \\
C_{G} & =\frac{\left|\Delta C_{l_{c i r c}}\right|\left(\sin \measuredangle\left(\Delta C_{l_{c i r c}}\right)-\frac{e_{c}}{c} 2 k \cos \measuredangle\left(\Delta C_{l_{c i r c}}\right)\right)}{c_{l_{\alpha}} \Delta \alpha G(k)\left[1+\left(\frac{e_{c}}{c} 2 k\right)^{2}\right]}
\end{aligned}
$$


Since we are interested in flutter problems encountered in cruise, given a cruising Mach number, the Reynolds number is fixed accordingly. Therefore, we do not seek an explicit Reynolds number dependence of the correction functions $C_{F}$ and $C_{G}$, as given by Eq. 22 and Eq. 23 above.

\section{CFD Methodology}

The correction method proposed in this study is based on a small set of steady state CFD simulations. The slope of the $\mathrm{Cl}-\alpha$ curve is required as an input to the correction method, which is provided by CFD. Additionally, to validate the new method, a number of unsteady CFD simulations were made for a range of reduced frequency, $k$, and Mach number, $M$. For this purpose, Reynolds-Averaged Navier-Stokes (RANS) solver, OVERFLOW, with the Spalart-Allmaras (SA) turbulence model ${ }^{14}$ is used. For the steady state, central differencing of inviscid terms in the Navier-Stokes equations and ARC3D diagonalized BeamWarming scalar pentadiagonal scheme $\mathrm{e}^{15,16}$ are used. For the unsteady simulations, HLLC upwind scheme along with the symmetric successive over-relaxation (SSOR) algorithm with subiterations are used.

\section{Grid and Time Stepsize Sensitivity}

Two computational grids for the NACA0012 airfoil were used, based on a prior grid sensitivity study ${ }^{17,18}$. For the unsteady simulations, various values of time stepsize were used. Figure 4(a,b) show the final selected

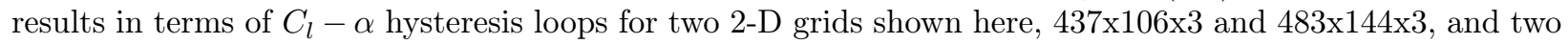
time stepsizes, $\Delta \tau=0.01$ and $\Delta \tau=0.001$; the former grid is referred to as the medium grid and the latter as the fine grid. Smooth results are demonstrated for the fine grid and $\Delta \tau=0.001$. Results for $\Delta \tau=0.01$ produce wiggles in the $C_{l}-\alpha$ hysteresis loop for both the grids. The medium grid still produces wiggles in the hysteresis loop for $\Delta \tau=0.001$, but the fine grid results in a smooth loop. So, the fine grid and $\Delta \tau=0.001$ are used for all unsteady simulations. The CFD results compare favorably with the experimental results ${ }^{19}$, as shown in Fig.4. Fine grid for the NACA0012 is shown in Fig. 5. Based on a previous study ${ }^{17,18}$, it turns out that the fine grid of $483 x 144 x 3$ and even finer grids ${ }^{17,18}$ yield practically the same result for unsteady non-pitching simulation. Therefore, the fine grid used here is taken to be an acceptable grid, especially because the kinks in the hysteresis loop disappear, whereas the medium grid (437x106x3) still produces small kinks.

\section{Results and Discussion}

The experiment was conducted at $M=0.755$ and $\mathrm{Re}=5.5 \times 10^{6}$. The experimental frequency of oscillation was $62.5 \mathrm{~Hz}$, and the pitching motion is defined by $\alpha=\bar{\alpha}+\alpha_{0} \sin (2 k \tau)$ where $\tau$ is the normalized time step, $\bar{\alpha}=0.016^{\circ}$ and $\alpha_{0}=2.51^{\circ}$. A set of CFD simulations corresponding to the experimental flight conditions was carried out. A check on the $C_{l}$ data from the CFD simulation in the frequency space produced an exact match with the experimental frequency of $62.5 \mathrm{~Hz}$. This is shown in Fig. 6a, and the corresponding time evolution of $C_{l}$ and the forcing history, $\alpha(\tau)$ is shown in Fig.6b.

With a good agreement between the results from the transonic test simulations and the experiment, as shown in Fig 4 and Fig. 6, we set out to compare our modified Theodorsen model with a number of simulations at $M=0.2,0.3,0.4,0.5,0.6,0.7$ and 0.755 . For each of these Mach numbers, a number of simulations were carried out corresponding to $k=0.02,0.04,0.06,0.08$ and 0.1 . Some simulations were made for $k=0.2,0.3,0.4$ and 0.5 also. Interesting details about phase inversion (also reported by Ref. 2) in the hysteresis plots were observed.

\section{Phase Inversion}

The results for $M=0.2$ are shown in Fig. $7(\mathrm{a}, \mathrm{b}, \mathrm{c})$. Phase inversion occurs at $k=0.2$, as shown in Fig $7 \mathrm{~b}$, where we see that the traverse of the hysteresis plot suddenly changes from counter-clockwise to clockwise at $k=0.2$. For lower values of reduced frequency, $k=0.02,0.04,0.06,0.08,0.1, C_{l}$ traverses counter-clockwise since the circulatory motion is the dominant component of the lift, and the phase of $C_{l}$ is negative. For $k$ $>0.1$, the $C_{l}$ phase is positive, i.e., the traverse of the hysteresis plot is clockwise, since the acceleration of the displaced fluid is higher and, therefore, the apparent mass contribution is higher and the phase of $C_{l}$ is 
positive. These effects were also observed in the CFD simulations ${ }^{5}$ with the focus on the thickness effects on the airfoil loads. In this study, we consider only NACA0012 and explore the Mach number effects on pitching airfoil loads. Similar behavior of $C_{l}$ is shown for $M=0.3,0.4$ and 0.5, as shown in Fig.8 through Fig.10. But, at $M=0.6$, the phase inversion occurs at $k=0.3$, as shown in Fig. 11, and it continues to $M$ $=0.7$ (Fig. 12). But, again, at $M=0.755$, the phase inversion now occurs at $k=0.5$, as shown in Fig.13.

\section{Validation of the Correction Method}

The proposed correction method modulates the Theodorsen model ${ }^{7}$ by coefficient functions, $C_{F}$ and $C_{G}$, (see Eqns, 22, 23 above). These are functions of reduced frequency, $k$, Mach number, $M$, and airfoil thickness, t. In the present study, we will focus on the dependence of these coefficient functions on $k$ and $M$ only. In a subsequent study, thickness effects will also be accounted for. The functions $C_{F}$ and $C_{G}$ are determined by Eqn. 22 and Eqn. 23 that need prescription of amplitude and phase shift of the circulatory lift, and these are provided by the steady state CFD computations for each value of $M$. Additionally, the corrected Theodorsen model is then validated by comparing the corresponding predictions with CFD predictions. As we shall see below, this comparison is very good all the way from incompressible to transonic range typical of cruising Mach number for transonic aircraft. Mach number from 0.2 to 0.755 were used for this validation and the results will be presented below. The accuracy of the proposed correction method is measured by how well its predictions agree with the CFD results, and the efficiency of the method is measured by how fast a $C_{l}-\alpha$ hysteresis loop is calculated relative to the CFD prediction, which turns out to be over 9 orders of magnitude faster, since the closed form solution provided by the method takes on the order of a milli-second while the CFD solution takes on the order of $72 \times 10^{6}$ sec.

Fig. 14 shows the variation the slope of the steady state $C_{l}-\alpha$ curve. At $M=0.2$, the slope is close to a value of $2 \pi$, as expected. Then, it begins to increase as shown in Fig. 14. The following results will be shown for a set of reduced frequencies, $k=0.02,0,04,0,06,0.08$ and 0.1 , which will be referred to as "various k". Fig. 15(a-e) shows variation of sinusoidally oscillating circulatory lift corresponding to various $k$ with time, $\tau$, for $M=0.2$. As shown, for lower values of $\mathrm{k}$, agreement between the CFD and the quasi-steady amplitudes of the circulatory lift is very good. Refreshing the definition of quasi-steady lift, it is that component of the lift that is produced according to the thin airfoil theory by the motion of the airfoil without the effect of the wake. This is expected since for lower frequency, lift is predominantly generated by the circulation around the airfoil (quasi-steady circulatory lift). As $k$ increases, differences between CFD and quasi-steady results become progressively distinguishable because of the added effect of the added mass (non-circulatory) effect. This frequency based variation is shown through Fig. 15a to Fig. 15e. The same trend in the circulatory amplitude persists at higher Mach numbers, as shown in Fig. 16 through Fig. 21, corresponding to $M=$ 0.3 through $M=0.755$, respectively.

The $C_{l}-\alpha$ hysteresis loops for $M=0.2$ for various $k$ are shown in Fig. 22(a-e) and the temporal evolution of $C_{l}$ with $\tau$ for various $k$ is shown in shown in Fig. 23(a-e). There is an excellent agreement between the CFD results and the corrected theoretical model, as shown in Fig. 22 and Fig. 23. The original Theodorsen model is shown to produce results in close agreement with the other predictions. This is expected since the original theoretical model holds strictly for incompressible flow.

For $M=0.3$, similar behavior for the $C_{l}-\alpha$ hysteresis loops and the temporal evolution of $C_{l}$ with $\tau$ is shown in Fig. 24 and Fig. 25, respectively. Since we are still in the incompressible range for $M=0.3$, for all practical purposes, results tend to agree with those for $M=0.2$. For $M=0.4$, some differences begin to surface in the $C_{l}-\alpha$ hysteresis loops and the temporal evolution of $C_{l}$ with $\tau$, especially higher frequencies, for reasons discussed above, as shown in Fig. 26 and Fig. 27, respectively. For $M=0.5,0.6,0.7$ and 0.755, the disagreement in the hysteresis loops becomes marked, again at higher frequencies. The results from CFD and the corrected model are in excellent agreement, but the original model incorrectly predicts, for obvious reasons, as shown in Fig. 28 through Fig. 35. For transonic Mach numbers, $M=0.6$ through $M=0.755$, all the frequencies except $k=0.02$ are incorrectly predicted by the original Theodorsen model. But, the corrected model predicts in excellent agreement with CFD.

Pressure distribution for various $k$ over NACA0012 airfoil is shown in Fig. 36(a,b). Fig. 36a corresponds to $M=0.7$ and Fig. 36b corresponds to $M=0.755$. There is a marked difference in the location of the shock between $M=0.7$ and $M=0.755$. For $M=0.7$, shock appears for $k=0.02,0.06$ and 0.1 , and the flow shows no discontinuities for $k=0.04$ and $k=0.08$. The location of shock is between $x=0.2$ and $x$ 
$=0.3$. But, for $M=0.755$, the shock is located around $x=0.4$, and, in addition, shock also appears for $k=0.04$ on the upper surface, and there is a cross-over in the pressure profile around $x=0.25$. The lower surface also shows a weak shock for $k=0.8$ around $x=0.2$.

Finally, with the corrected Theodorsen method validated with CFD, we present the correction functions (Eq. 22 and Eq. 23), $C_{F}$ and $C_{G}$, in Fig. 37 and Fig. 38. Fig. 37a and Fig. 37b show the variation of $C_{F}$ and $C_{G}$ with $M$, respectively, with $k$ as a parameter. As $M$ becomes small $C_{F}$ and $C_{G}$ tend to a value of 1 , thereby recovering Theodorsen's original model. Similarly, Fig. 38a and Fig. 38b show the variation of $C_{F}$ and $C_{G}$ with $k$, respectively, with $M$ as a parameter.

\section{Concluding Remarks}

A new method has been proposed that extends the classical Theodorsen's theory of unsteady aerodynamics for incompressible flow [7] to transonic flow. The method proposes a modified Theodorsen's function to correct for changes in the amplitude and phase shift of the circulatory lift due to transonic flow. The modified function captures the effect of reduced frequency, $k$, and Mach number, $M$, as applicable to transonic flow. For the present study, NACA0012 airfoil was considered. The method is partly based on the results from the steady state CFD simulations at different Mach numbers, Various values of $M$ from 0.2 through 0.755 and $k$ from 0.02 through 0.1, typical of transonic flutter problems, were used to validate the new method with the CFD predictions for this $M$ and $k$ range. The new method gives results in very good agreement with the CFD predictions. The modulation functions that modify the original Theodorsen functions are taken to be functions of $M$ and $k$. In an upcoming study, these modulation functions will also be treated as functions of airfoil thickness such as to enable us to study the transonic flutter problem of Variable Camber Continuous Trailing Edge Flap (VCCTEF) for a NASA generic transport model (GTM). The goal is to devise a state space representation of the VCCTEF in transonic flutter and an associated transfer function that will readily be used in the study of aeroelasticity problems of interest.

\section{Acknowledgements}

This work was partially funded by the Advanced Air Transport Technology Project under the Advanced Air Vehicles Program of the NASA Aeronautics Research Mission Directorate (ARMD). The authors also would like to acknowledge Boeing Research and Technology and the University of Washington for their collaboration with NASA under NASA contract NNL11AA05B task order NNL12AD09T entitled "Development of Variable Camber Continuous Trailing Edge Flap System for B757 Configured with a More Flexible Wing." OVERFLOW 2.2 was used in the present work for which the authors would like to thank Pieter Buning of NASA Langley Research Center.

\section{References}

[1] Albano, E. and Rodden, W., "A Doublet-Lattice Method for Calculating Lift Distributions on Oscillating Surfaces in Subsonic Flows", AIAA Journal, Vol. 7, No. 2, February 1969, pp. 279-285.

[2] Bartels, R. E. and Stanford, B. K., "Aeroelastic Optimization with an Economical Transonic Flutter Constraint Using Naviertokes Aerodynamics," J. Aircraft, Vol. 55, No. 4, Julyugust 2018

[3] Mallik, W., Schetz, J. A. and Kapania, R. K., "Rapid Transonic Flutter Analysis for Aircraft Conceptual Design Applications," AIAA J., Vol. 56, No. 6, June 2018

[4] Max M. J. Opgenoord, Max M. J., Drela, M. and Willcox, K. E., "Physics-Based Low-Order Model for Transonic Flutter Prediction," AIAA J., Vol. 56, No. 4, April 2018

[5] Motta, V., Guardone, A. and Giuseppe, Q., "Influence of airfoil thickness on unsteady aerodynamic loads on pitching airfoils," J. Fluid Mech., Vol. 774, 2015, pp. 460-487 
[6] Choi, J., Colonius, T. and Williams, D. R., "Surging and plunging oscillations of an airfoil at low Reynolds number," J. Fluid Mech., vol. 763, 2015, pp. 237-253. doi:10.1017/jfm.2014.674

[7] Theodorsen, T., "General Theory of Aerodynamic Instability and the mechanism of Flutter", NACA Report No. 496, 1949.

[8] Buning, P. G., "NASA OVERFLOW Overset Grid CFD Flow Solver,ASA Langley Research Center, Hampton, VA; 2016, https://overflow.larc.nasa.gov

[9] Buning, P. G., Gomez, R. J., and Scallion, W. I., 2004, "CFD Approaches for Simulation of Wing-Body Stage Separation,IAA Paper No. 2004-4838.

[10] Kandula, M., and Buning, P. G., "Implementation of LU-SGS Algorithm and Roe Upwinding Scheme in OVERFLOW Thin-Layer Navier-Stokes Code,IAA Paper No. 94-2357, 1994.

[11] Kaul, U. K., "Do large structures control their own growth in a mixing layer ? An assessment," J. Fluid Mech., vol. 190, 1988, pp. 427-450

[12] Nguyen, N. and Ting, E., "Inertial Force Coupling to Nonlinear Aeroelasticity of Flexible Wing Aircraft," AIAA Dynamics Specialists Conference, AIAA-2016-1094, January 2016

[13] Bisplinghoff, R., Ashley, H., and Halfman, R., Aeroelasticity, Dover Publications, Inc., Mineola, New York, 1996, pp. 261-262.

[14] Spalart, P. R., and Allmaras, S. R., "A One-Equation Turbulence Model for Aerodynamic Flows," AIAA 92-0439, AIAA 30th Aerospace Sciences Meeting and Exhibit, Reno, NV , January 1992.

[15] Beam, R., and Warming, R. F., 1976, "An Implicit Finite-Difference Algorithm for Hyperbolic Systems in Conservation Law Form,. Comp. Phys., 22(1), pp. 8710.

[16] Pulliam, T. and Chaussee, D. R., 1976, "A Diagonal Form of an Implicit Approximate-Factorization Algorithm," J. Comp. Phys., vol. 39, no. 2,1981, pp. 347-363. ISSN 0021-9991

[17] Kaul, U. K. and Nguyen, N. T., "Drag Optimization Study of Variable Camber Continuous Trailing Edge Flap (VCCTEF) Using OVERFLOW," AIAA 2014-2444, 32nd Applied Aerodynamics Conference, Atlanta, GA, June 2014

[18] Kaul, U. K. and Nguyen, N. T., "Drag Characterization Study of Variable Camber Continuous Trailing Edge Flap," ASME J. Fluids Eng., Oct. 2018, Vol. 140, pp. 101108-1

[19] Landon, R. H., "NACA 0012. Oscillatory and transient pitching flap,", AGARD-R-702, Compendium of Unstead Aerodynamic Measuremtents, ISBN 92-835-I430. 0 


\section{Figures}
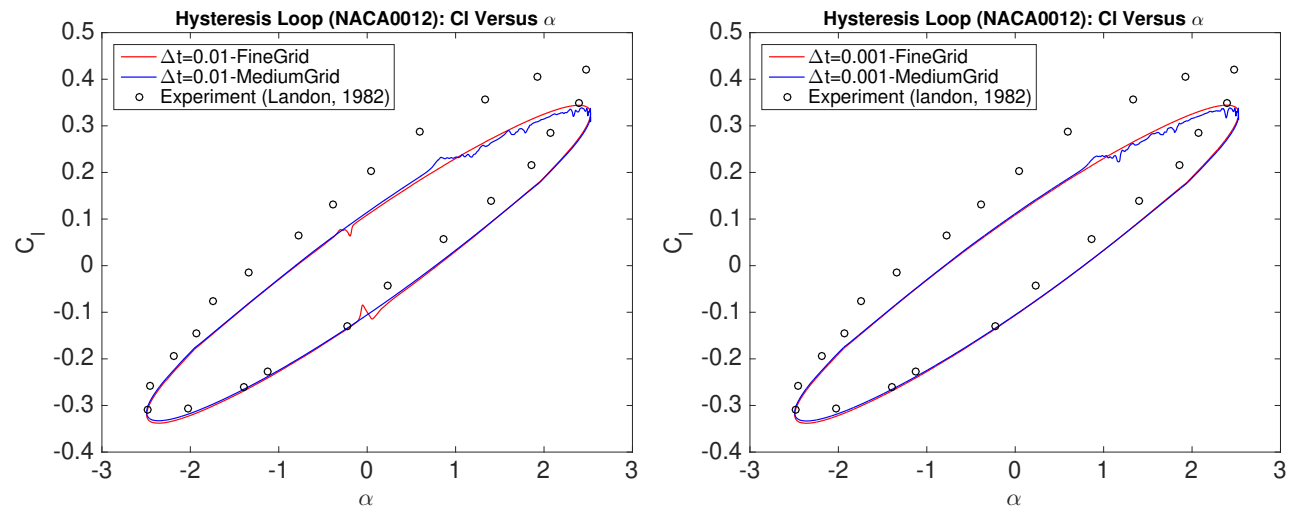

Figure 4: $C_{l}-\alpha$ hysteresis loops

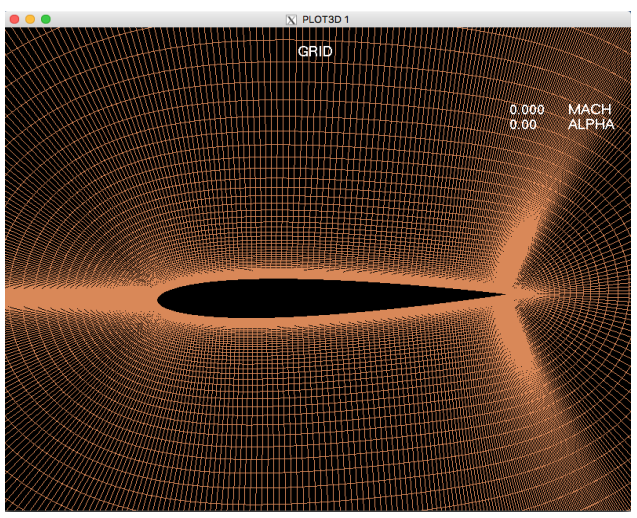

Figure 5: Fine grid for the NACA0012

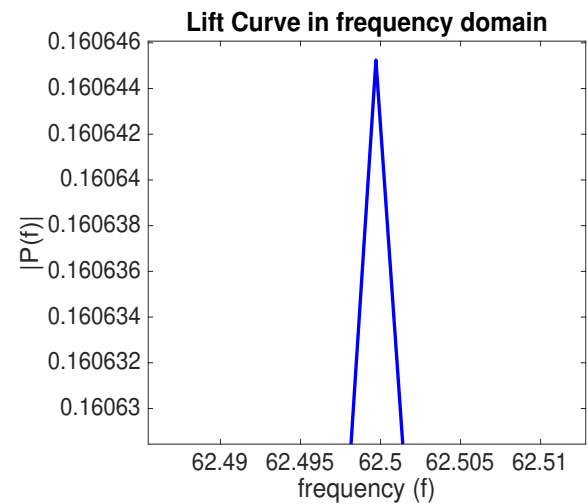

(a) Power spectrum of $C_{l}$



(b) Time evolution of $C_{l}$ with forcing, $\alpha(\tau)$

Figure 6: Exact match of experimental and CFD frequency data; experiment: $62.5 \mathrm{~Hz}$ 


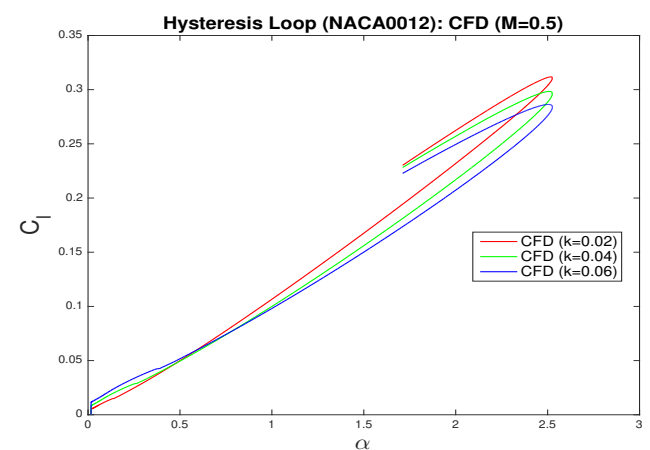

(a) First cycle $(\mathrm{k}=0.02,0.04,0.06)$

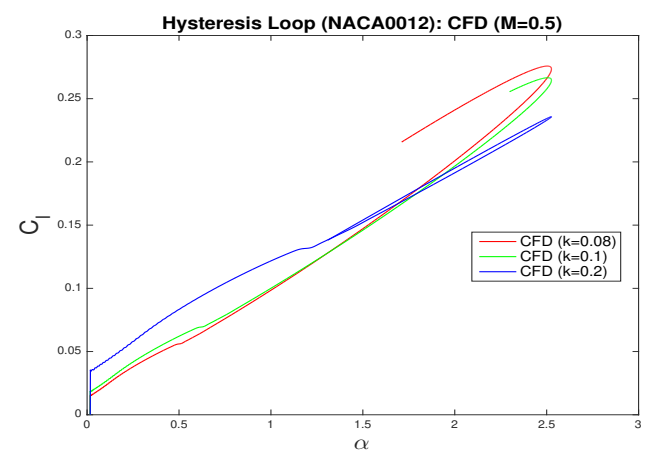

(b) First cycle $(\mathrm{k}=0.08, \mathrm{k}=0.1, \mathrm{k}=0.2)$

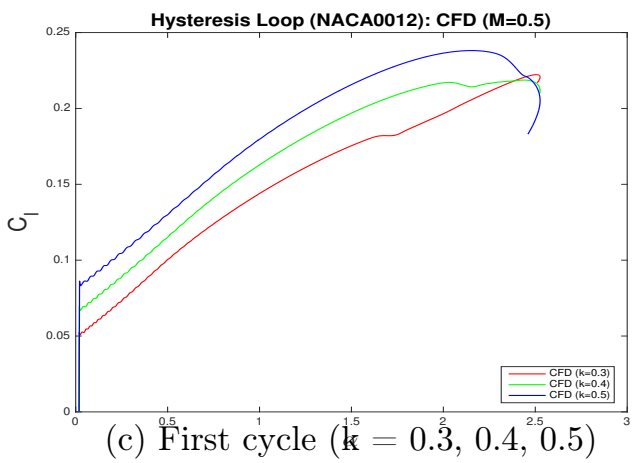

Figure 10: Phase inversion occurs at $\mathrm{k}=0.2$ for $\mathrm{M}=0.5$

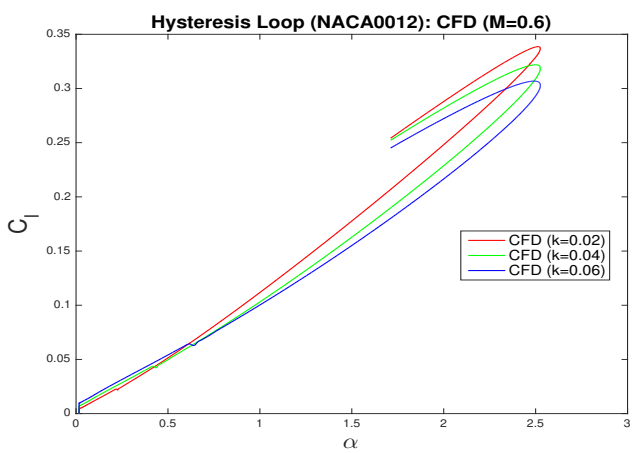

(a) First cycle $(\mathrm{k}=0.02,0.04,0.06)$

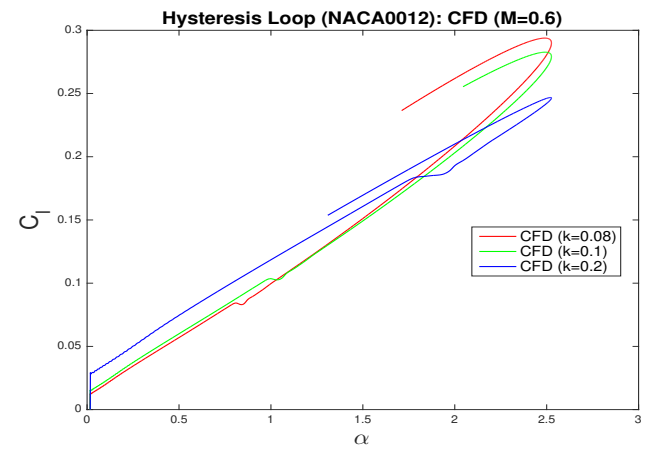

(b) First cycle $(\mathrm{k}=0.08, \mathrm{k}=0.1, \mathrm{k}=0.2)$

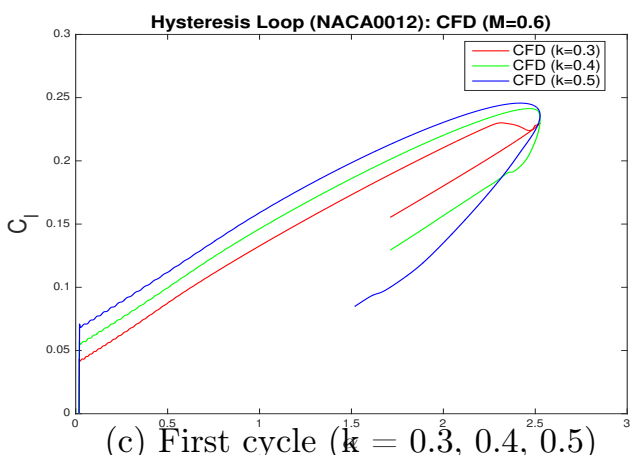

Figure 11: Phase inversion occurs at $\mathrm{k}=0.3$ for $\mathrm{M}=0.6$ 


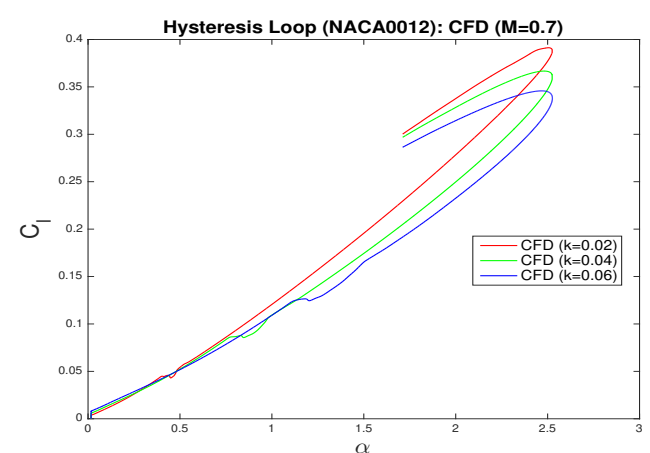

(a) First cycle $(\mathrm{k}=0.02,0.04,0.06)$

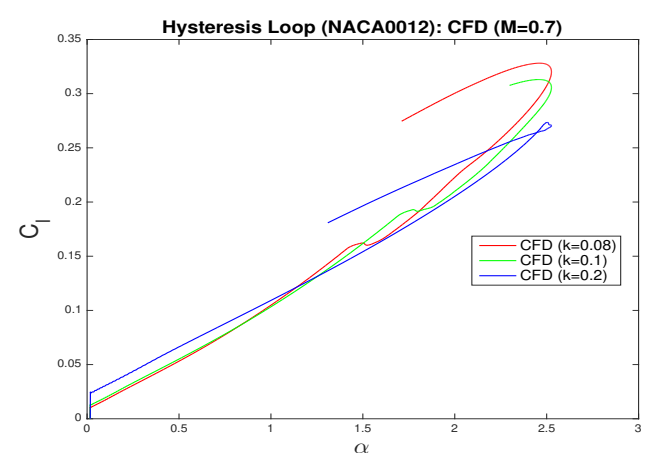

(b) First cycle $(\mathrm{k}=0.08, \mathrm{k}=0.1, \mathrm{k}=0.2)$

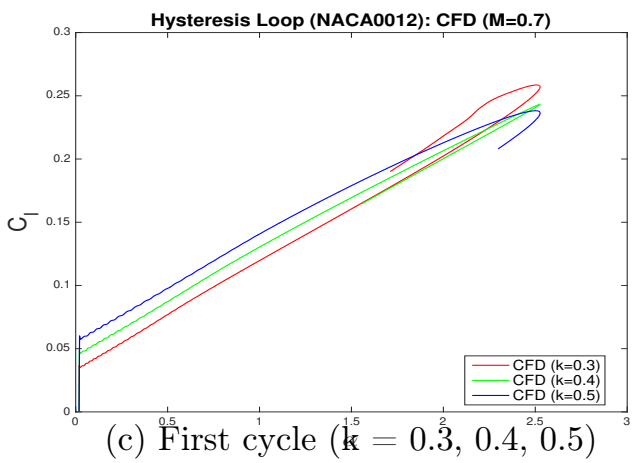

Figure 12: Phase inversion occurs at $\mathrm{k}=0.4$ for $\mathrm{M}=0.7$

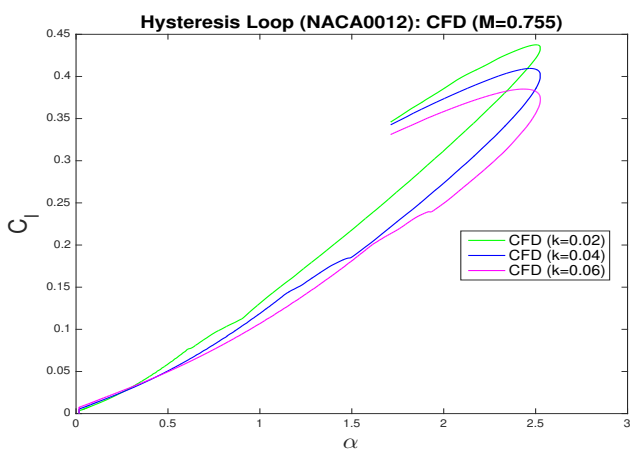

(a) First cycle $(\mathrm{k}=0.02,0.04,0.06)$

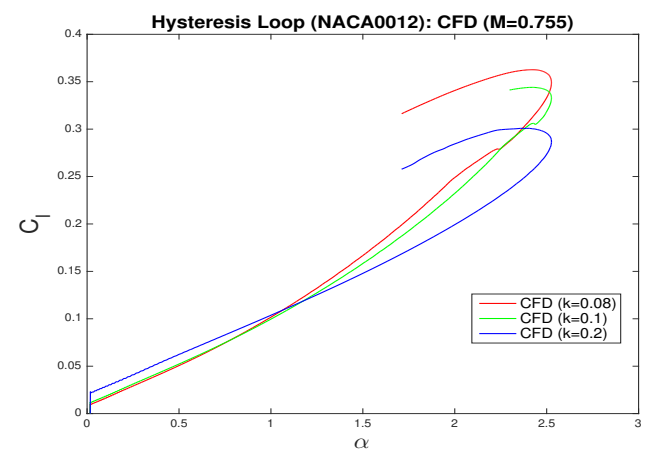

(b) First cycle $(\mathrm{k}=0.08, \mathrm{k}=0.1, \mathrm{k}=0.2)$



Figure 13: Phase inversion occurs at $\mathrm{k}=0.5$ for $\mathrm{M}=0.755$ 


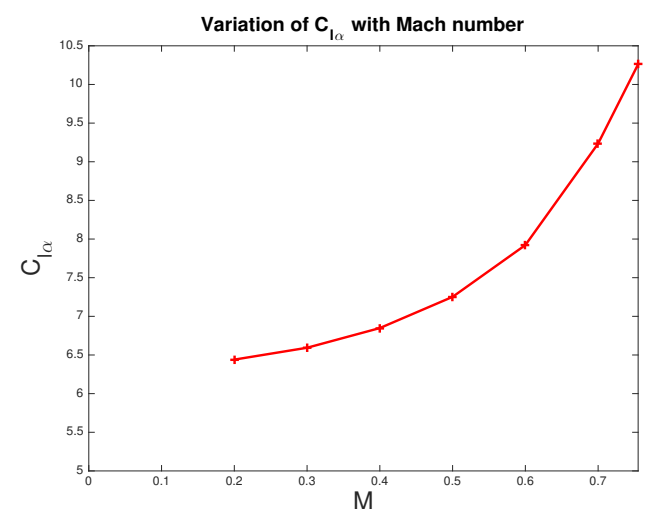

Figure 14: $C_{l \alpha}$ vs M 


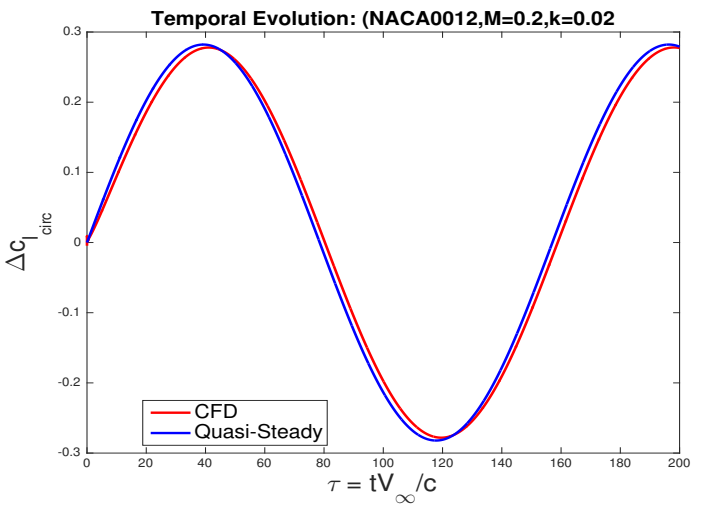

(a) $\mathrm{k}=0.02$

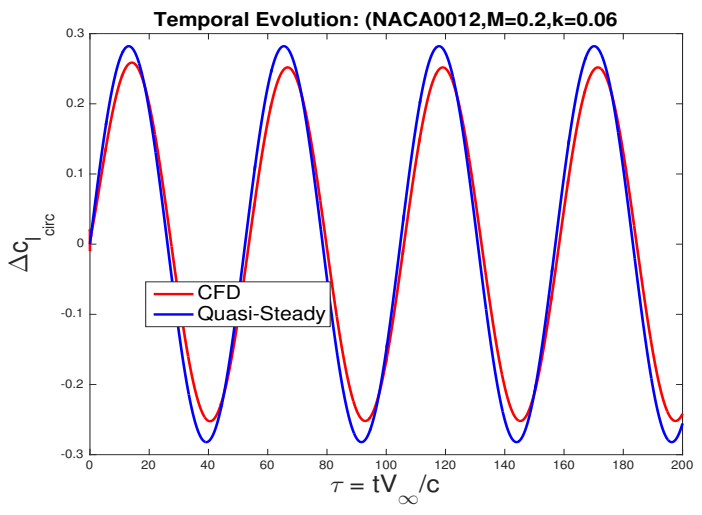

(c) $\mathrm{k}=0.06$

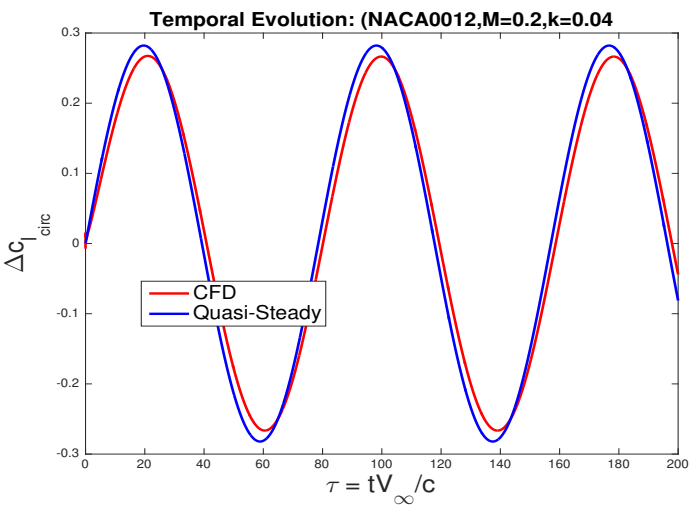

(b) $\mathrm{k}=0.04$

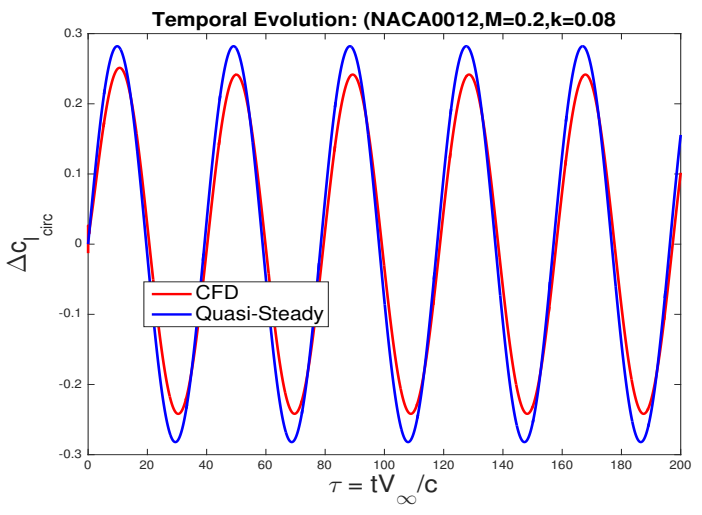

(d) $\mathrm{k}=0.08$

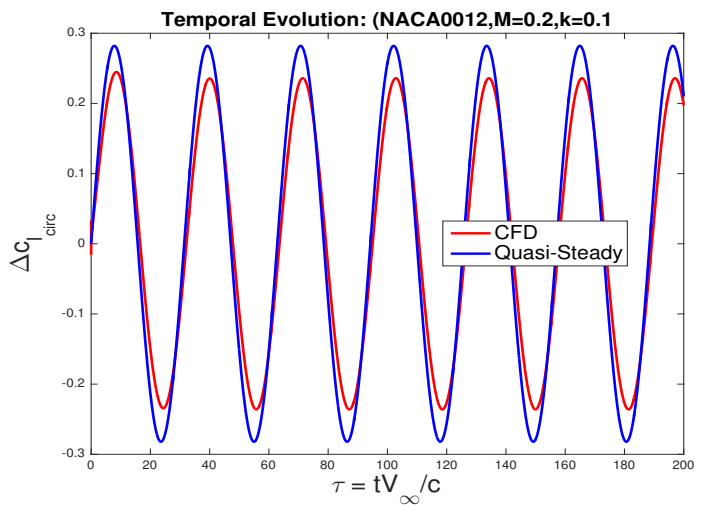

(e) $\mathrm{k}=0.1$

Figure 15: Circulatory lift: $\mathrm{M}=0.2$ 


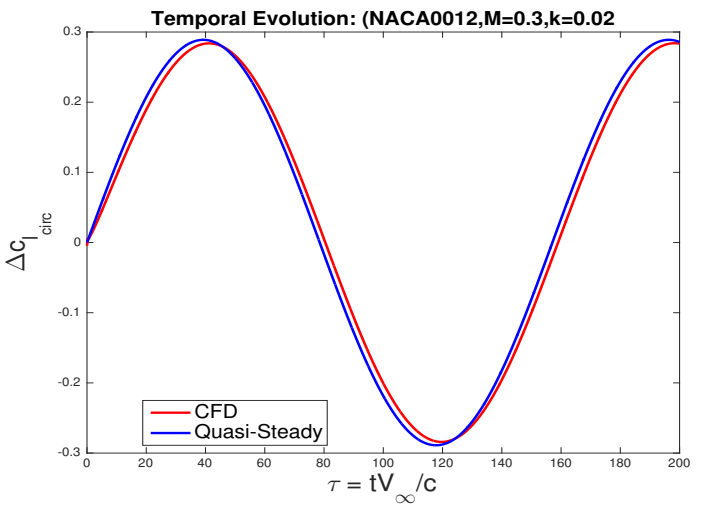

(a) $\mathrm{k}=0.02$

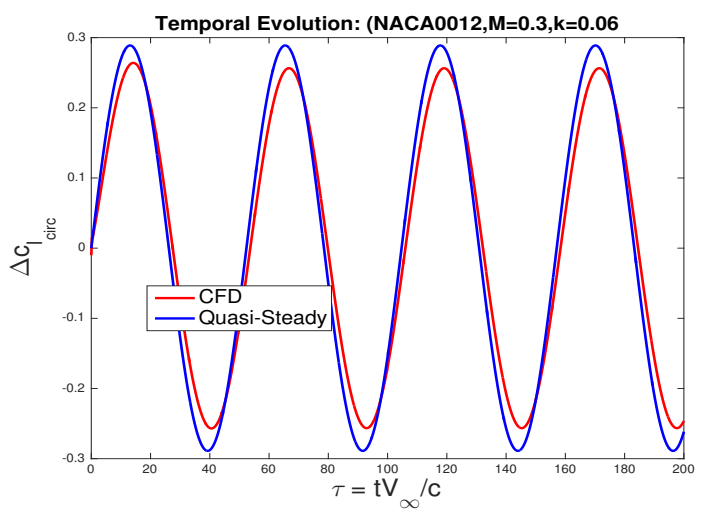

(c) $\mathrm{k}=0.06$

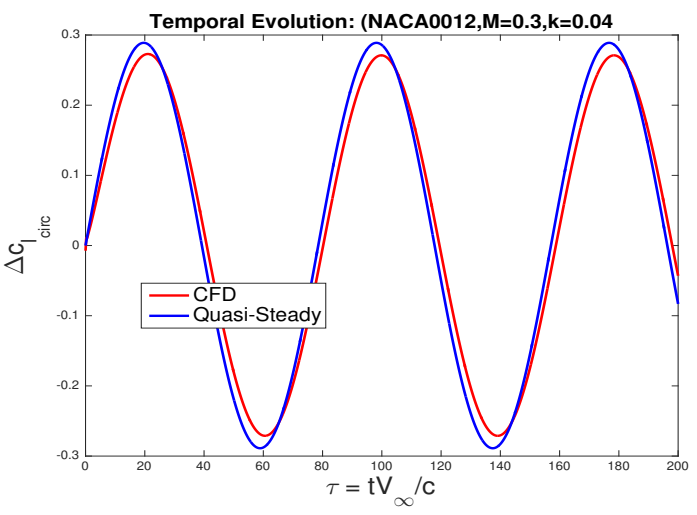

(b) $\mathrm{k}=0.04$

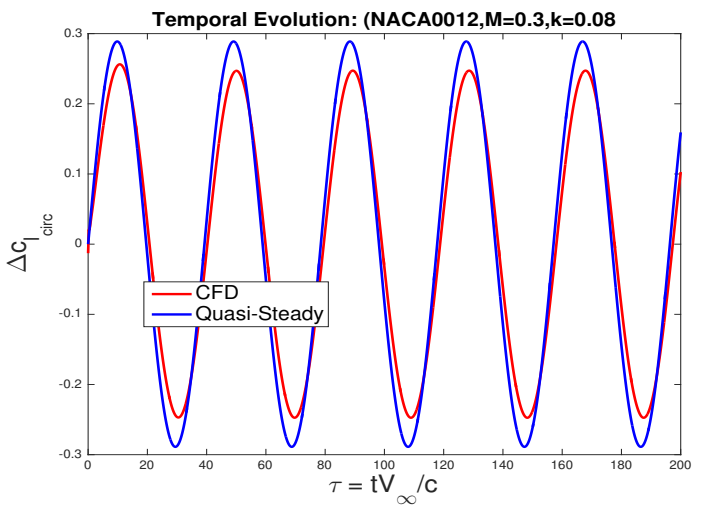

(d) $\mathrm{k}=0.08$

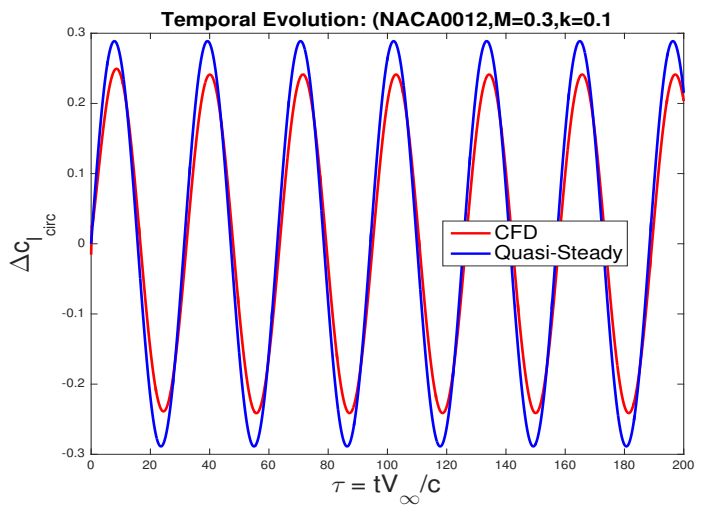

(e) $\mathrm{k}=0.1$

Figure 16: Circulatory lift: $\mathrm{M}=0.3$ 


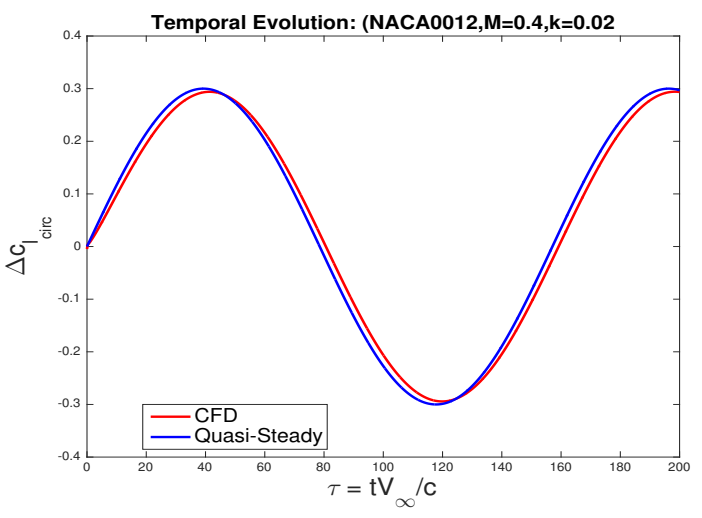

(a) $\mathrm{k}=0.02$

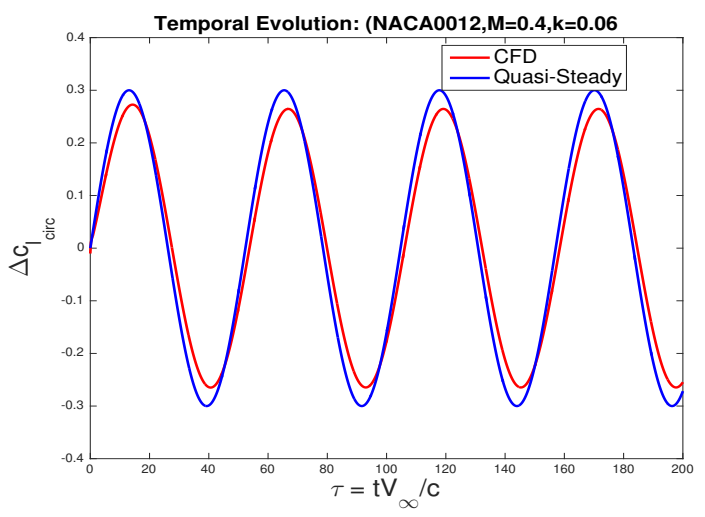

(c) $\mathrm{k}=0.06$

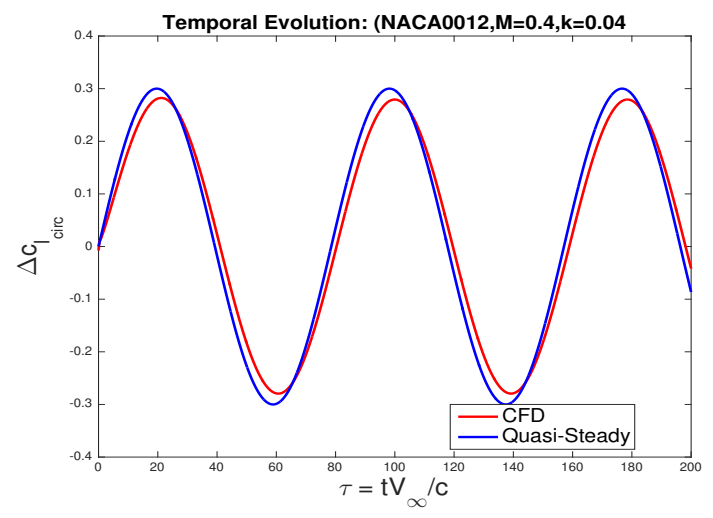

(b) $\mathrm{k}=0.04$

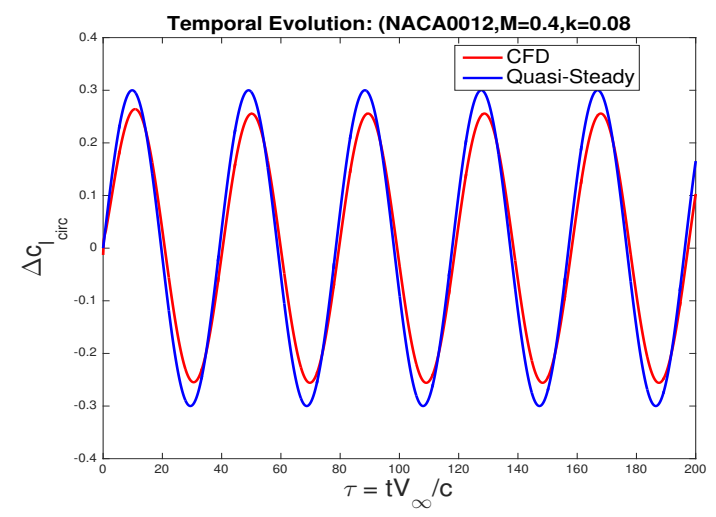

(d) $\mathrm{k}=0.08$

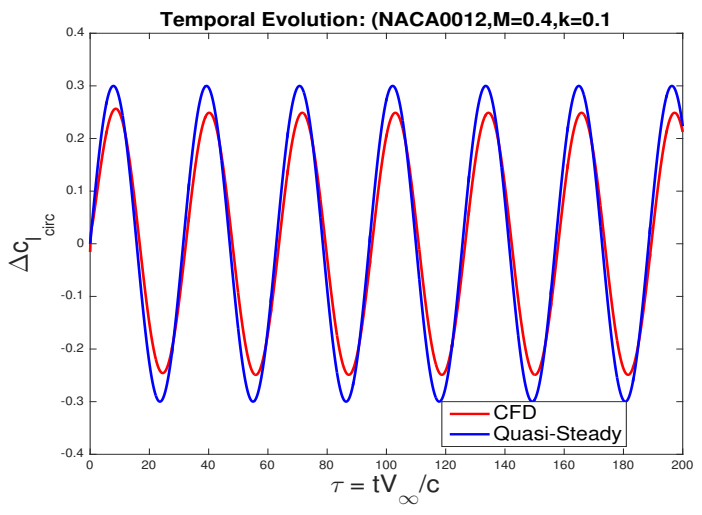

(e) $\mathrm{k}=0.1$

Figure 17: Circulatory lift: $\mathrm{M}=0.4$ 


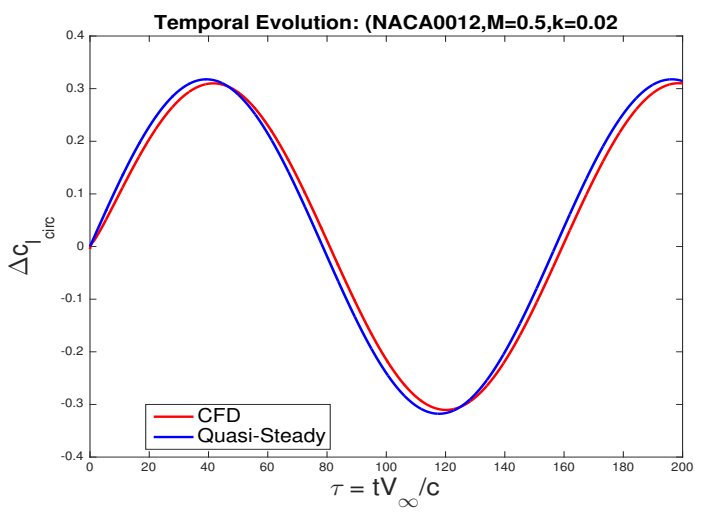

(a) $\mathrm{k}=0.02$

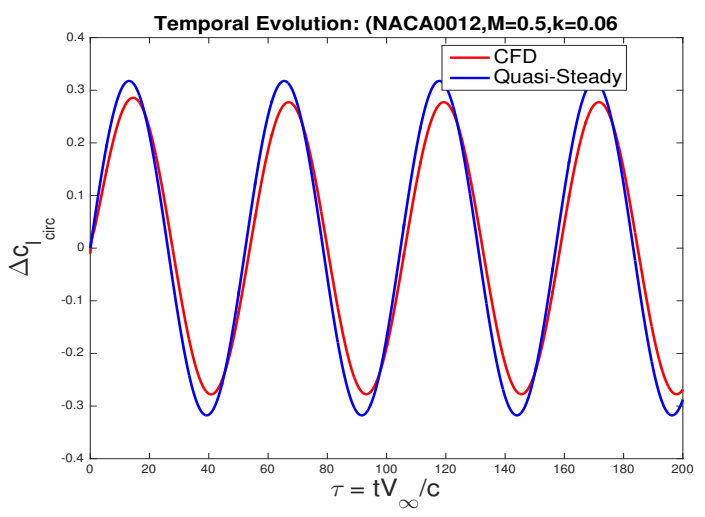

(c) $\mathrm{k}=0.06$



(b) $\mathrm{k}=0.04$

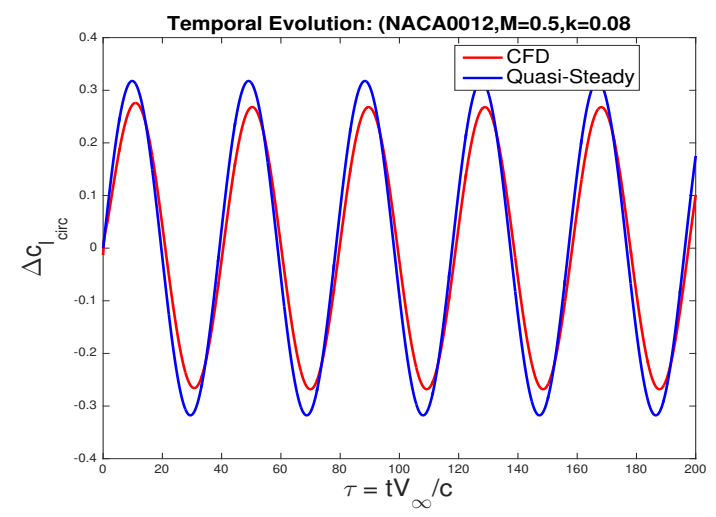

(d) $\mathrm{k}=0.08$

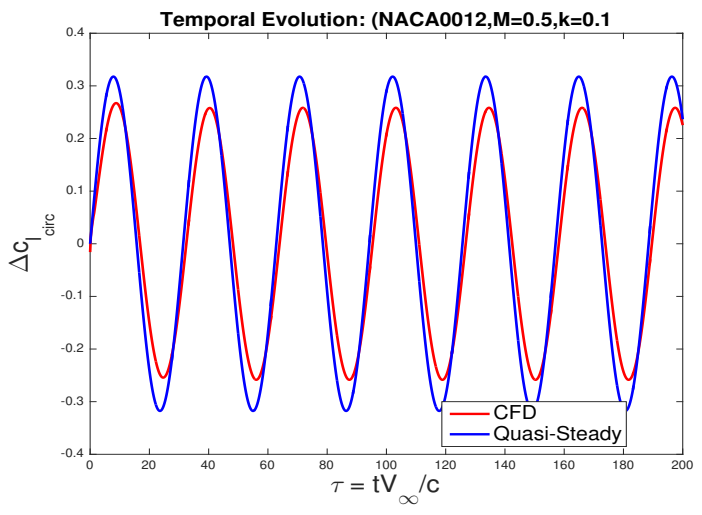

(e) $\mathrm{k}=0.1$

Figure 18: Circulatory lift: $\mathrm{M}=0.5$ 


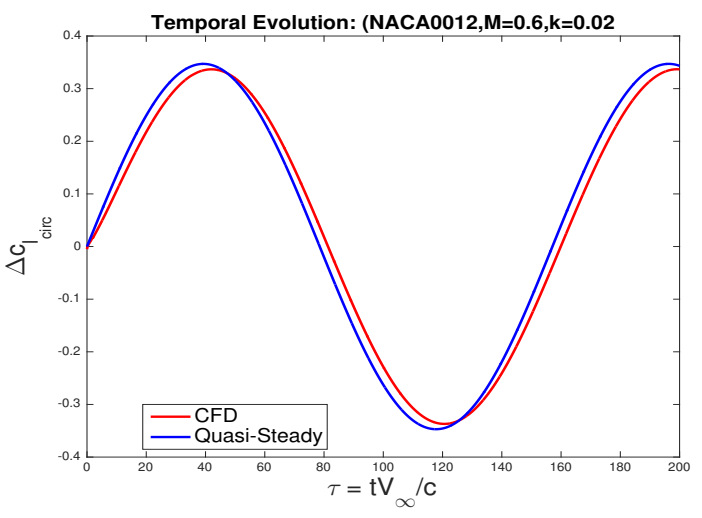

(a) $\mathrm{k}=0.02$

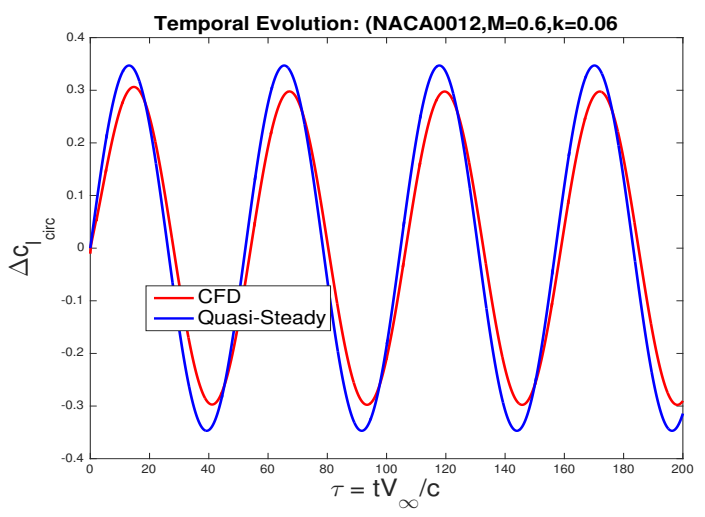

(c) $\mathrm{k}=0.06$

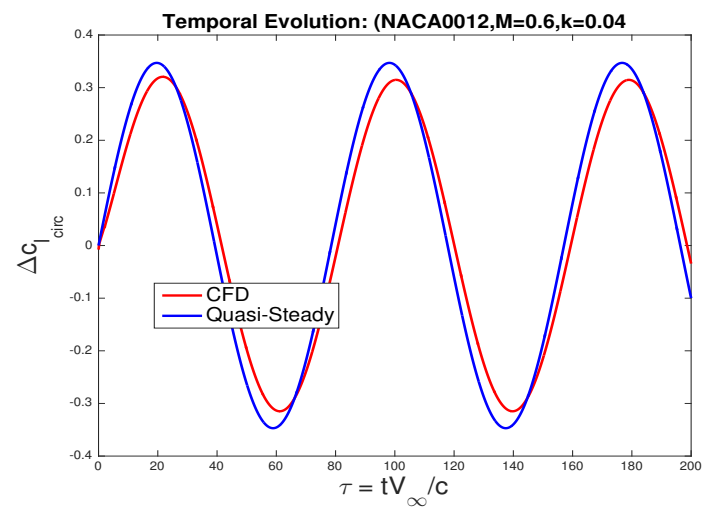

(b) $\mathrm{k}=0.04$

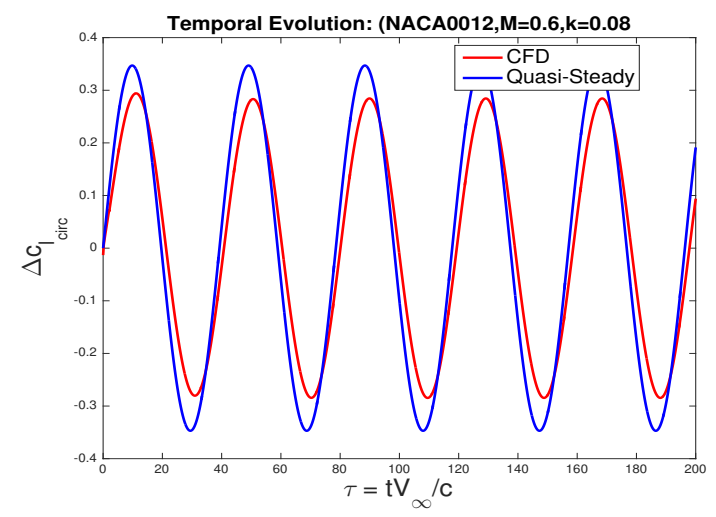

(d) $\mathrm{k}=0.08$

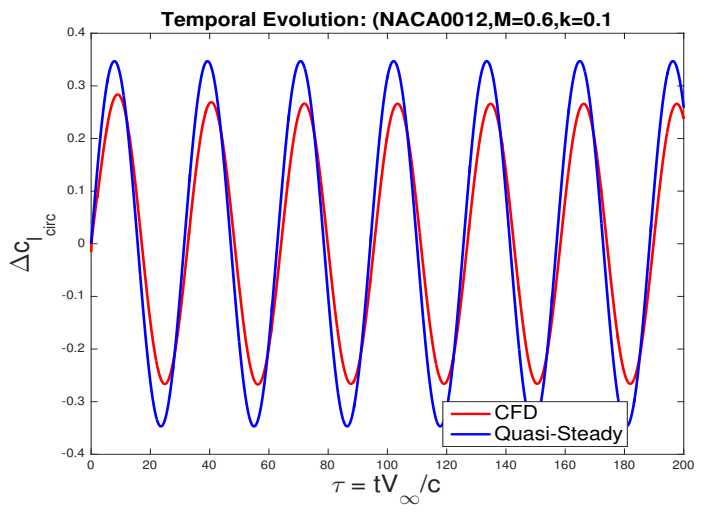

(e) $\mathrm{k}=0.1$

Figure 19: Circulatory lift: $M=0.6$ 


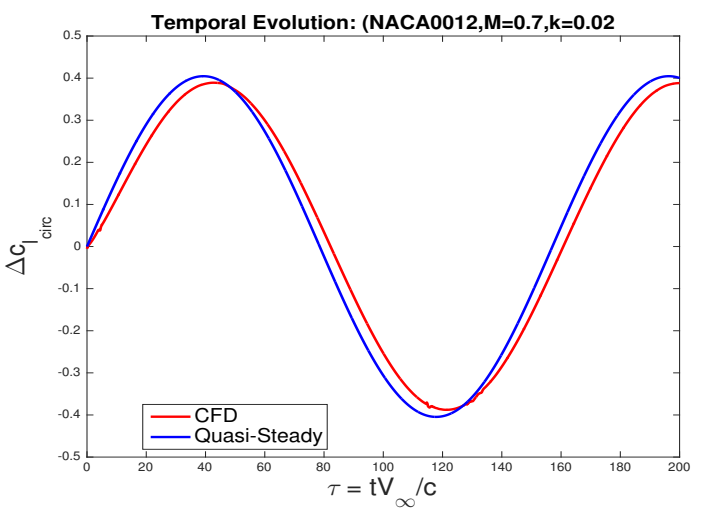

(a) $\mathrm{k}=0.02$

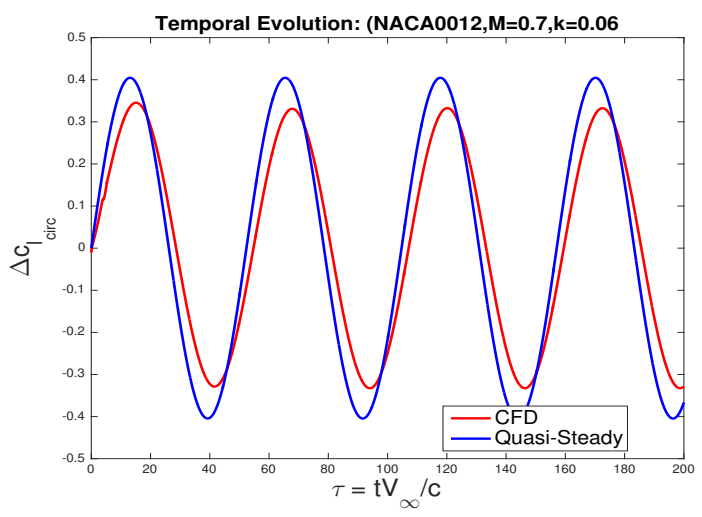

(c) $\mathrm{k}=0.06$

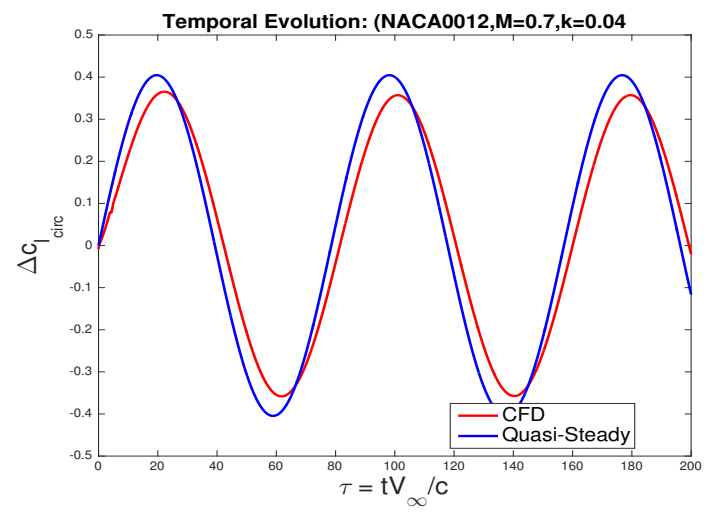

(b) $\mathrm{k}=0.04$

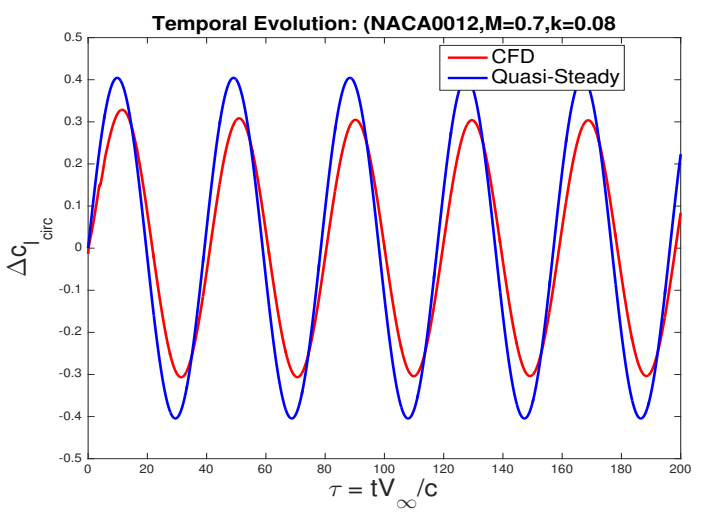

(d) $\mathrm{k}=0.08$

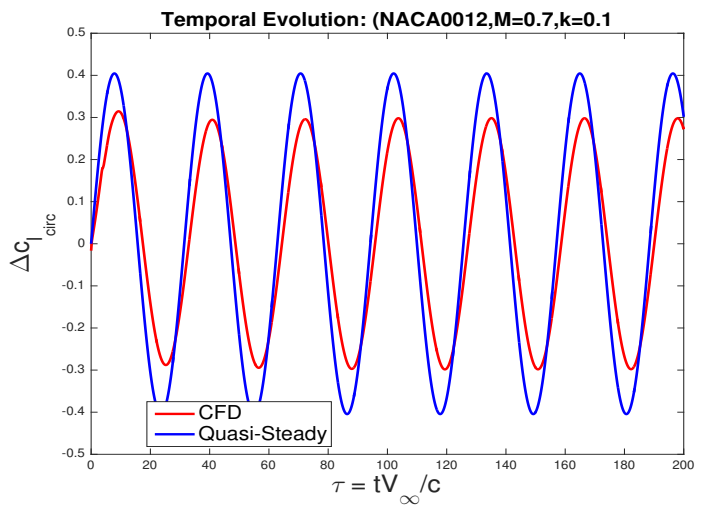

(e) $\mathrm{k}=0.1$

Figure 20: Circulatory lift: $M=0.7$ 


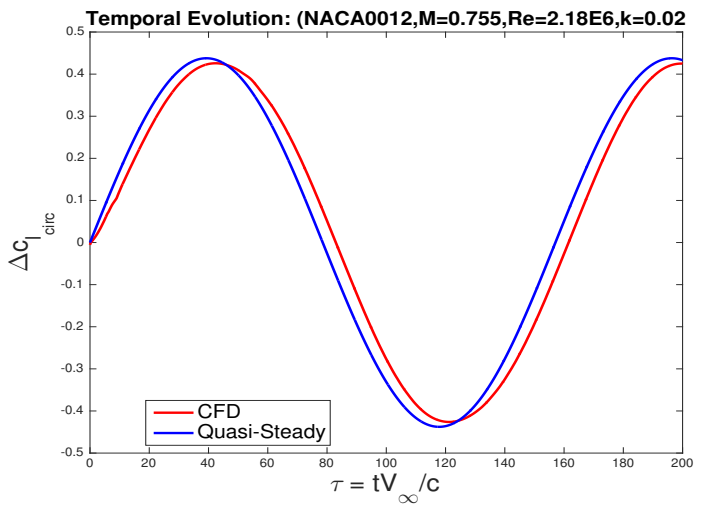

(a) $\mathrm{k}=0.02$

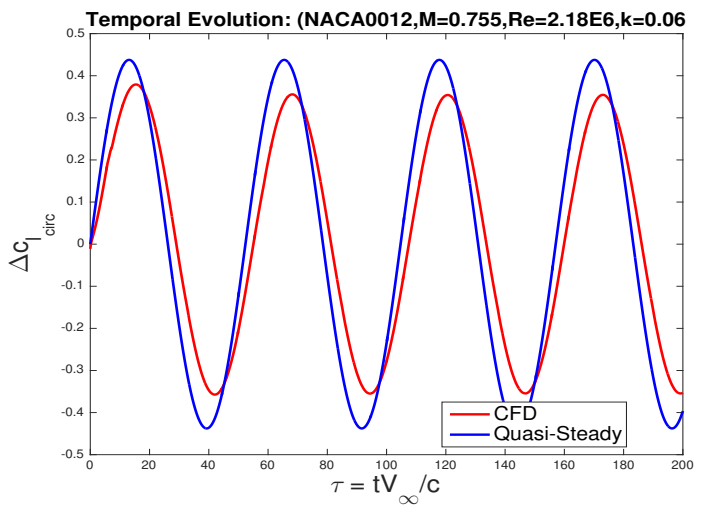

(c) $\mathrm{k}=0.06$

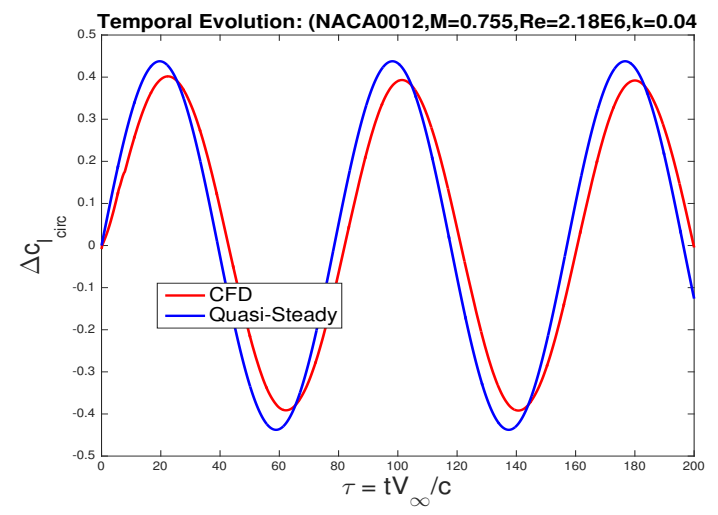

(b) $\mathrm{k}=0.04$

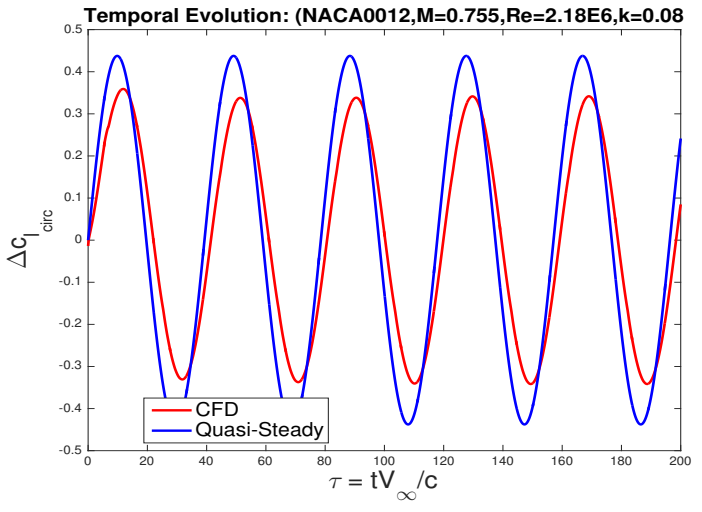

(d) $\mathrm{k}=0.08$

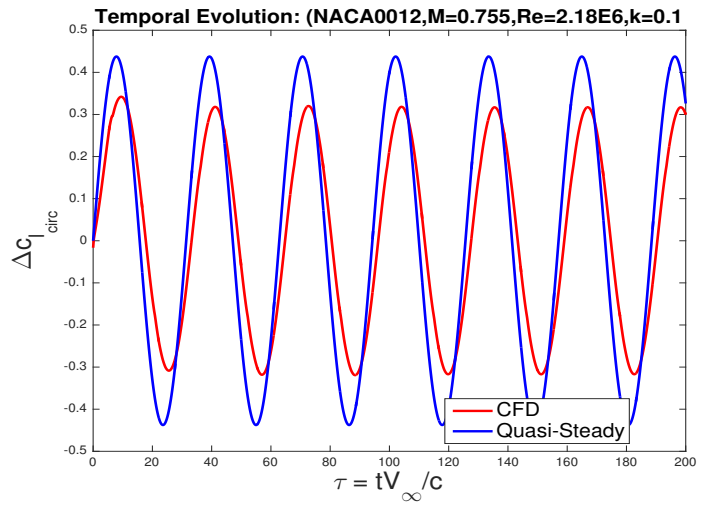

(e) $\mathrm{k}=0.1$

Figure 21: Circulatory lift: $M=0.755$ 


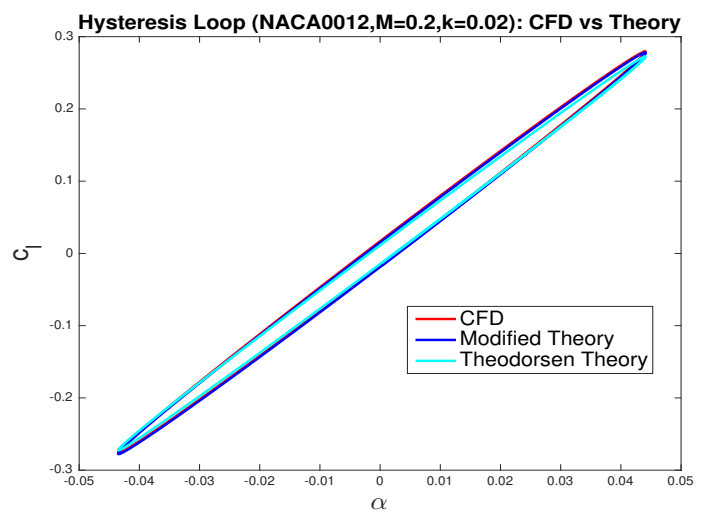

(a) $\mathrm{k}=0.02$

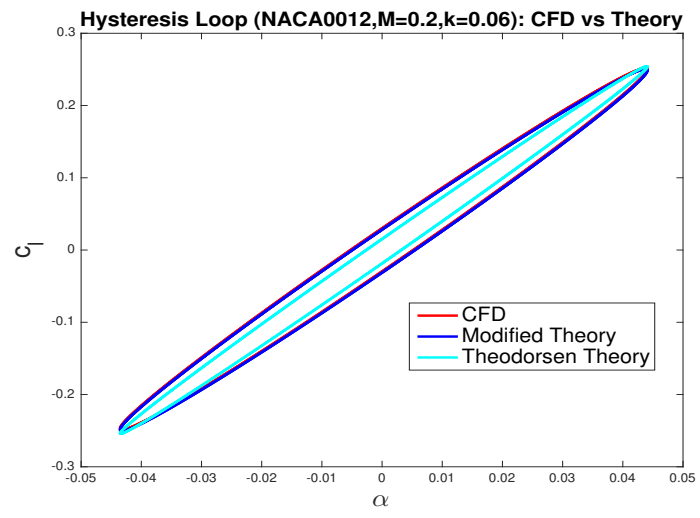

(c) $\mathrm{k}=0.06$

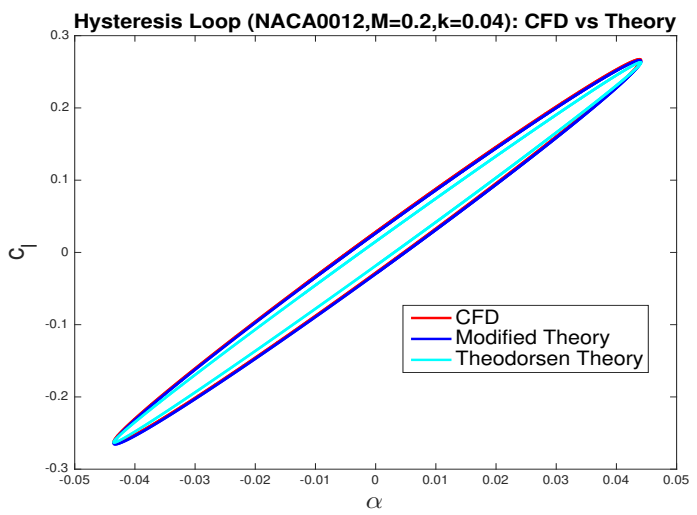

(b) $\mathrm{k}=0.04$

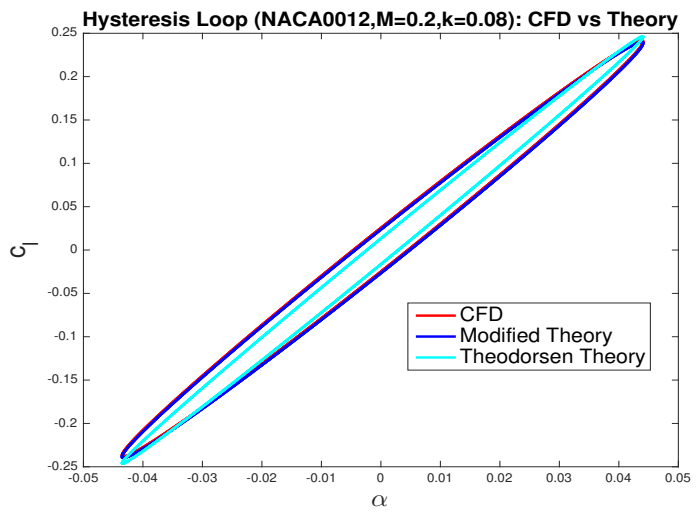

(d) $\mathrm{k}=0.08$

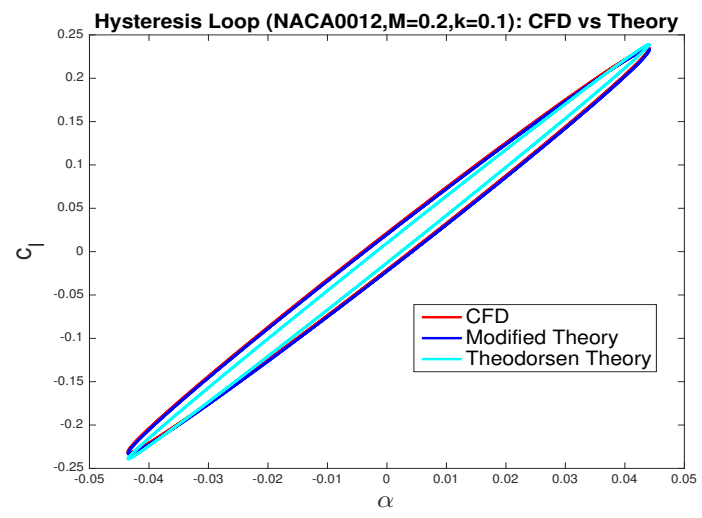

(e) $\mathrm{k}=0.1$

Figure 22: $C_{l}-\alpha$ hysteresis loop: $\mathrm{M}=0.2$ 


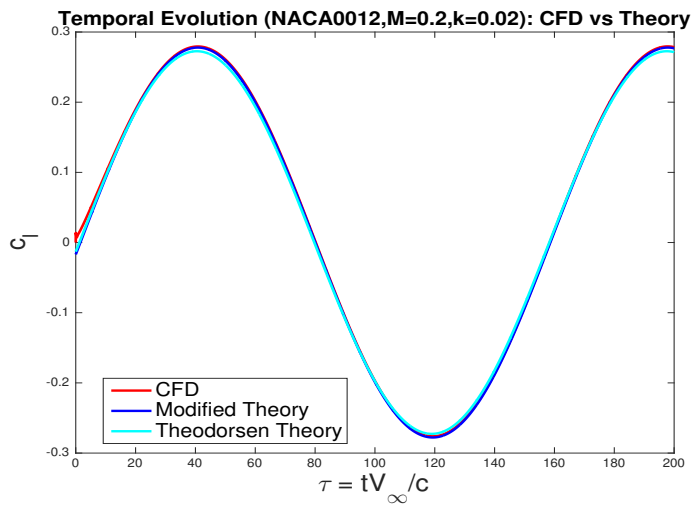

(a) $\mathrm{k}=0.02$

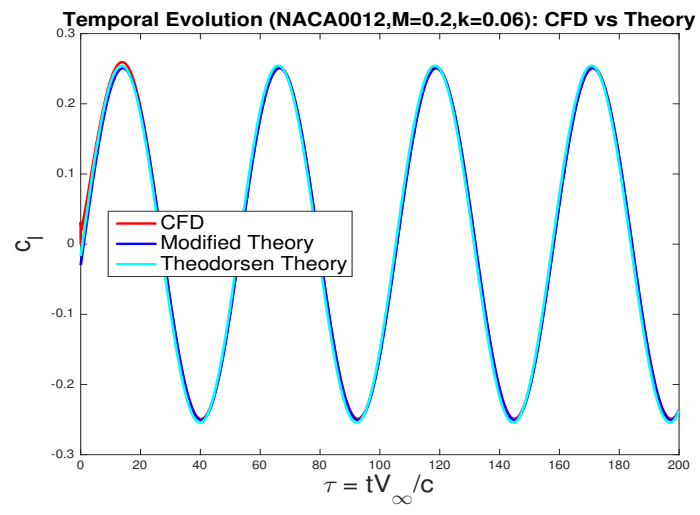

(c) $\mathrm{k}=0.06$

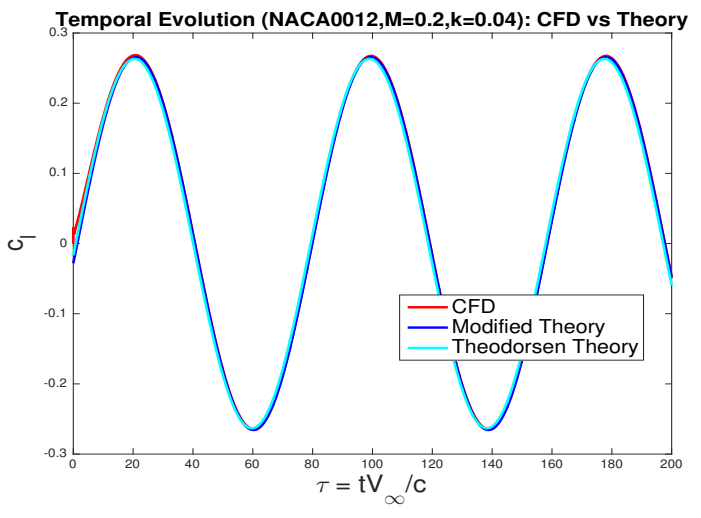

(b) $\mathrm{k}=0.04$

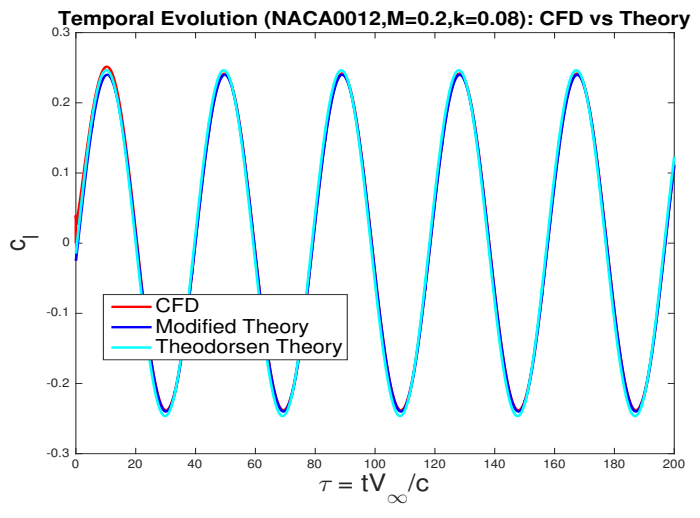

(d) $\mathrm{k}=0.08$

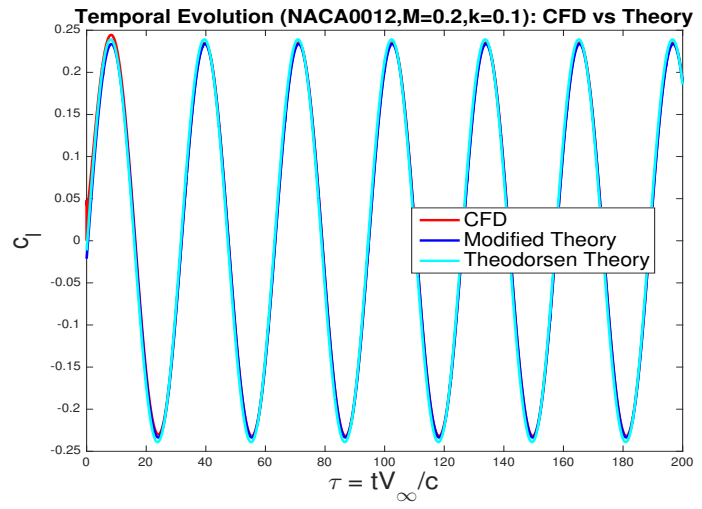

(e) $\mathrm{k}=0.1$

Figure 23: $C_{l}$ vs $\tau: \mathrm{M}=0.2$ 


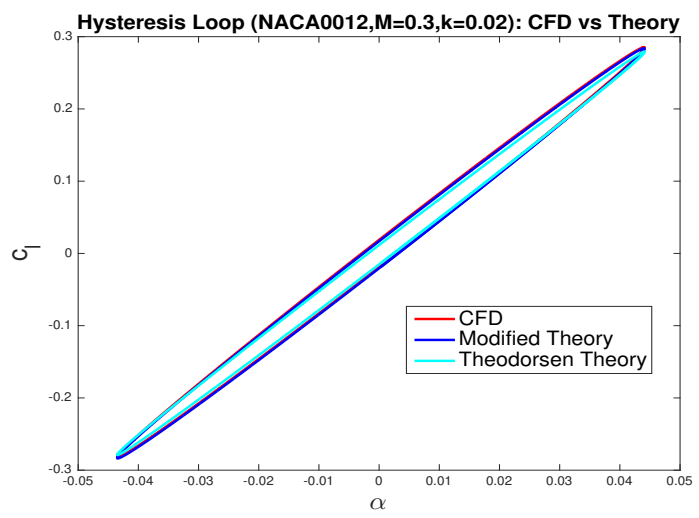

(a) $\mathrm{k}=0.02$

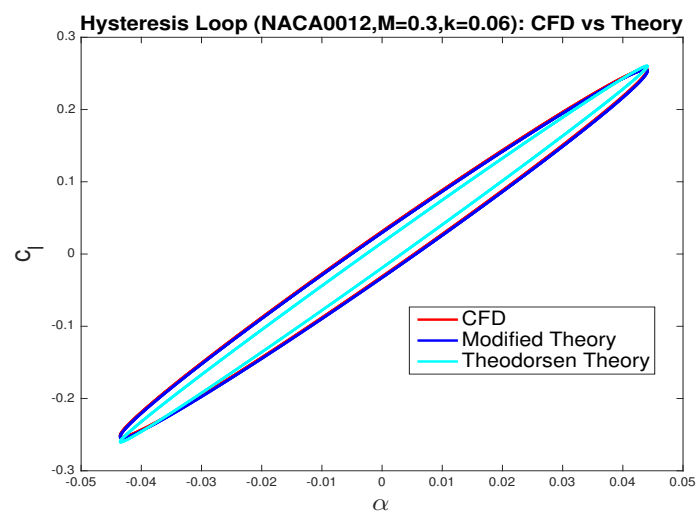

(c) $\mathrm{k}=0.06$

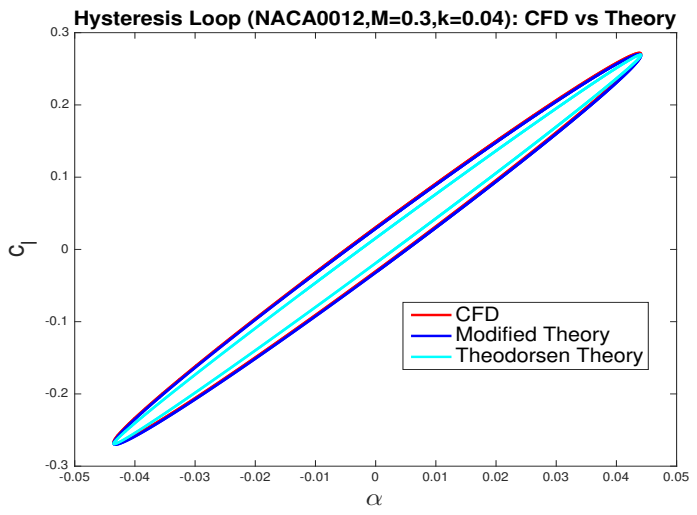

(b) $\mathrm{k}=0.04$

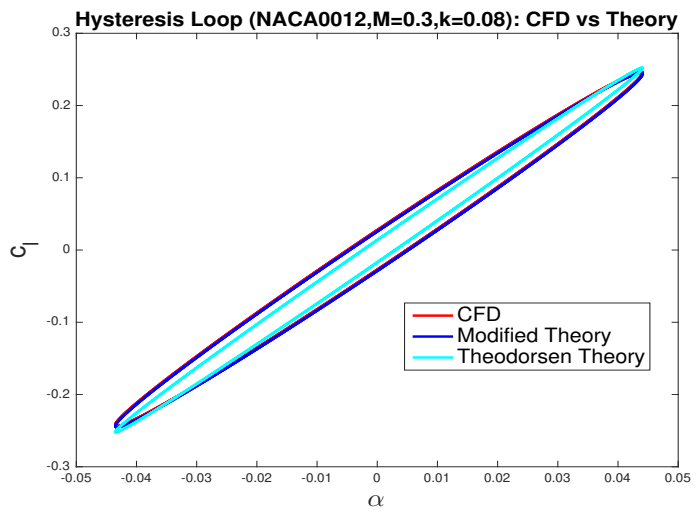

(d) $\mathrm{k}=0.08$

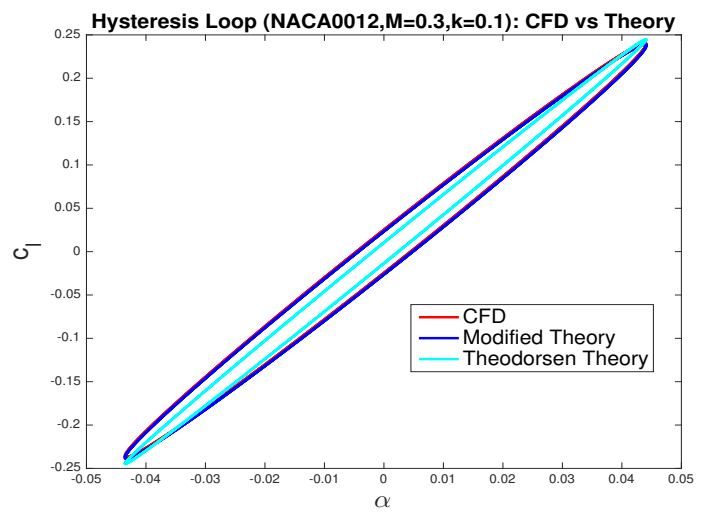

(e) $\mathrm{k}=0.1$

Figure 24: $C_{l}-\alpha$ hysteresis loop: $\mathrm{M}=0.3$ 


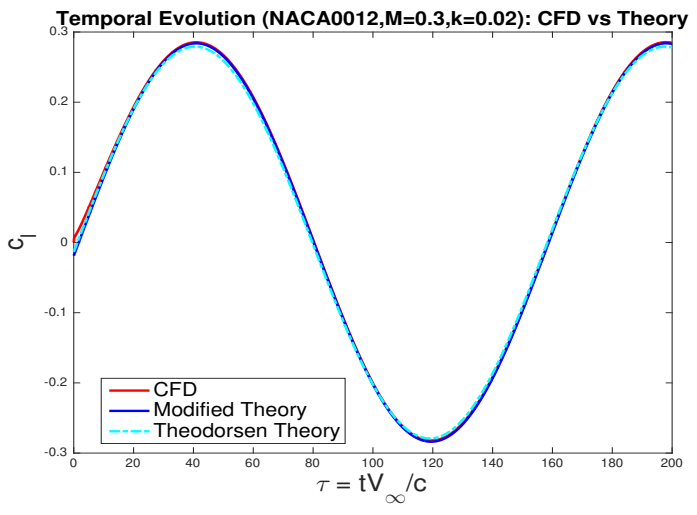

(a) $\mathrm{k}=0.02$

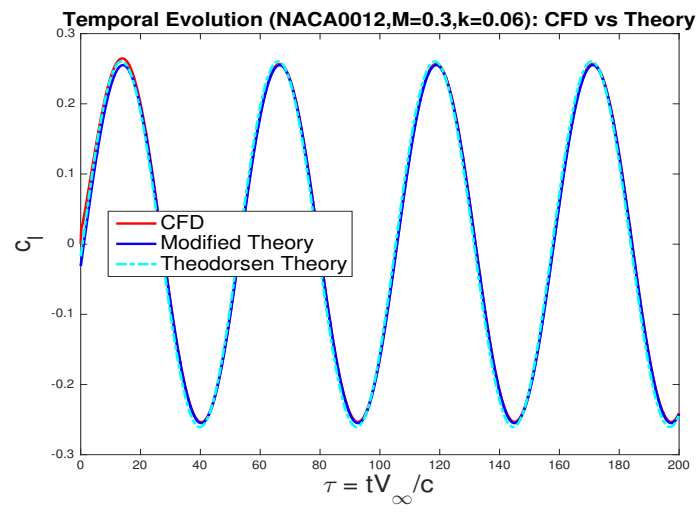

(c) $\mathrm{k}=0.06$

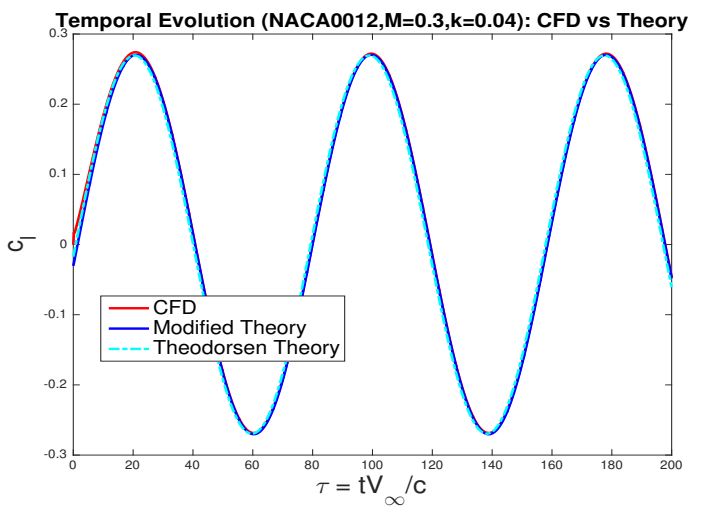

(b) $\mathrm{k}=0.04$

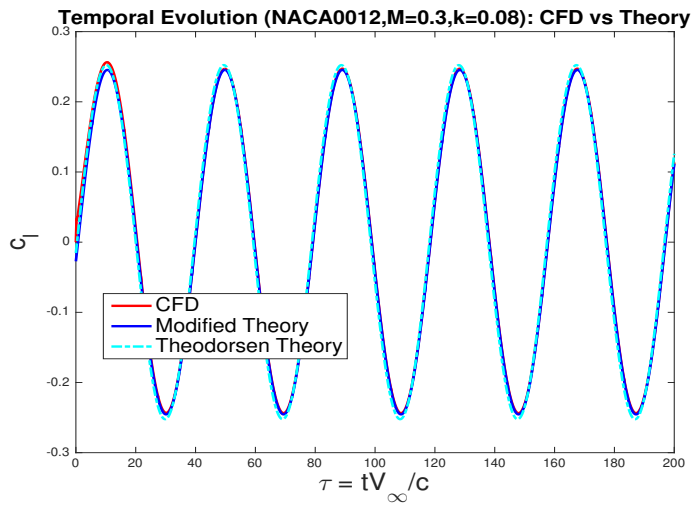

(d) $\mathrm{k}=0.08$

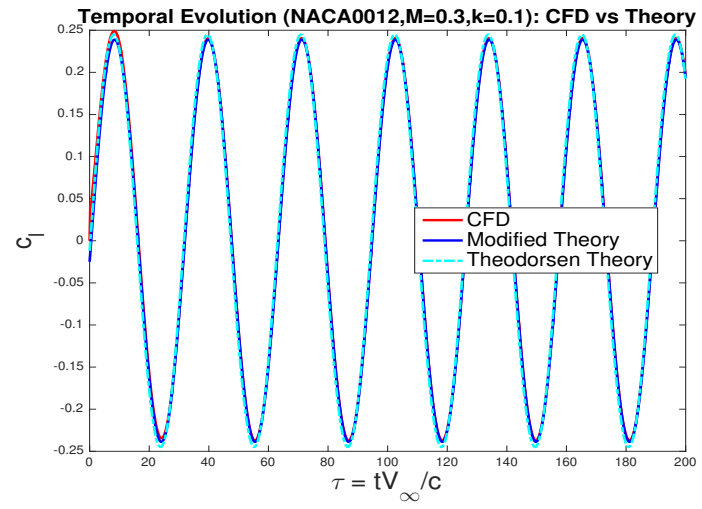

(e) $\mathrm{k}=0.1$

Figure 25: $C_{l}$ vs $\tau: \mathrm{M}=0.3$ 


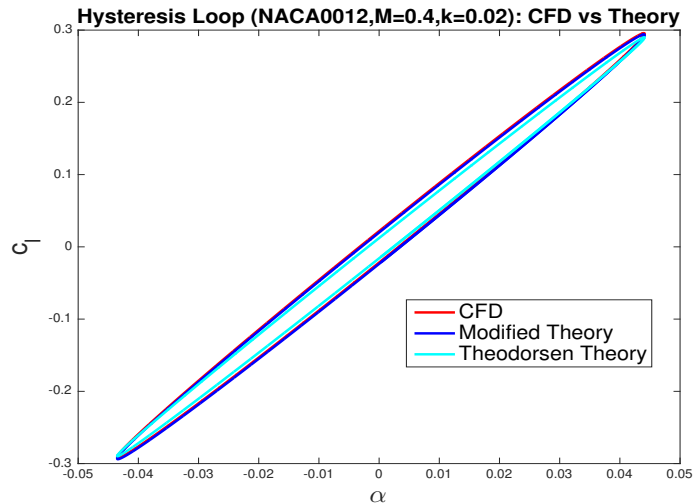

(a) $\mathrm{k}=0.02$

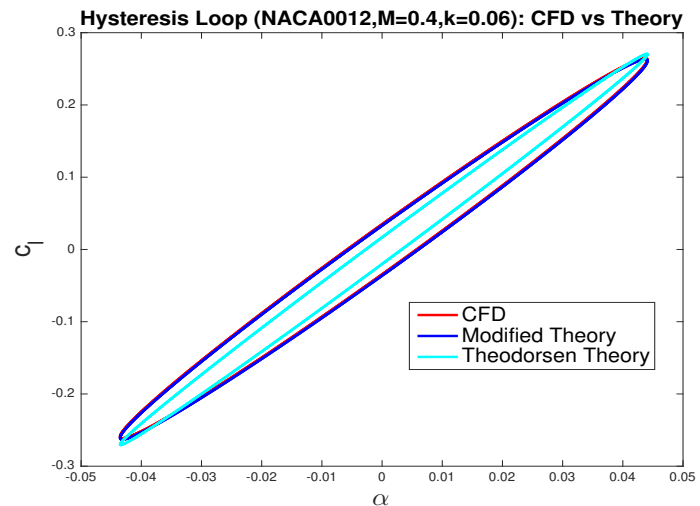

(c) $\mathrm{k}=0.06$

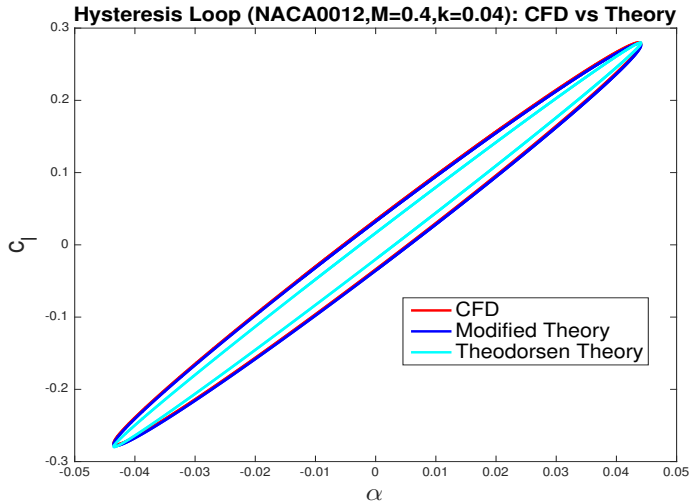

(b) $\mathrm{k}=0.04$

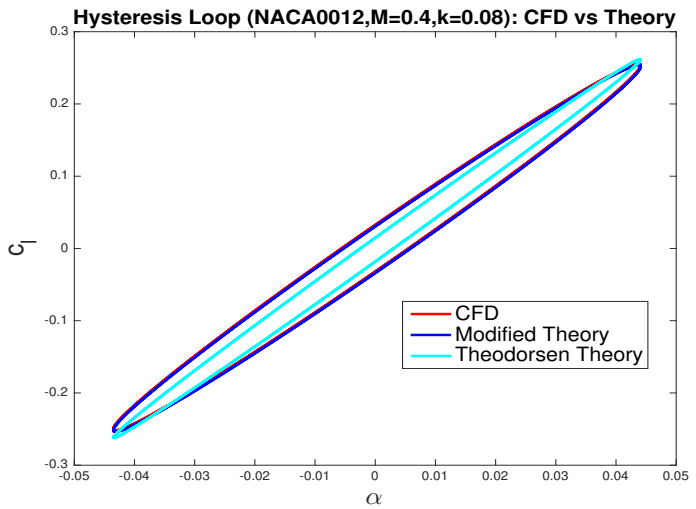

(d) $\mathrm{k}=0.08$

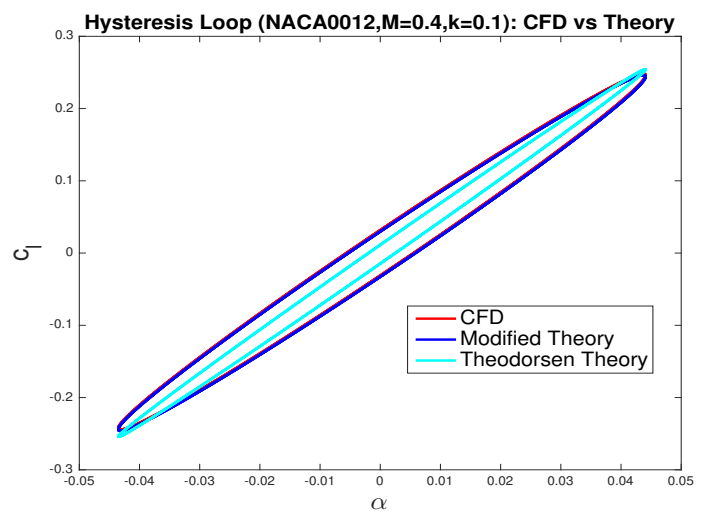

(e) $\mathrm{k}=0.1$

Figure 26: $C_{l}-\alpha$ hysteresis loop: $\mathrm{M}=0.4$ 


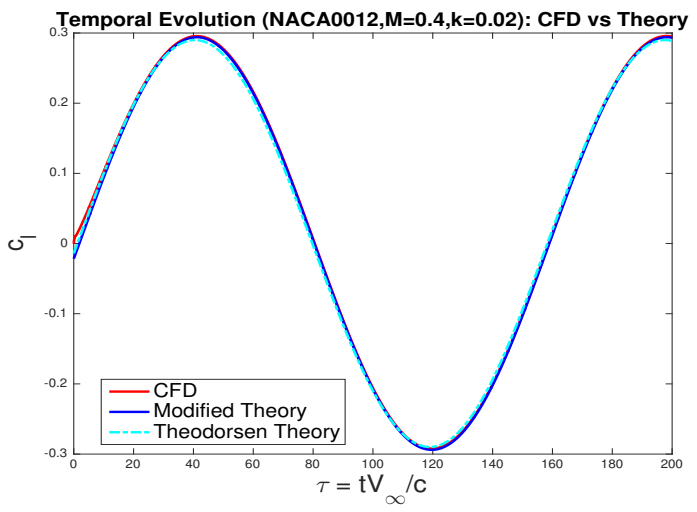

(a) $\mathrm{k}=0.02$

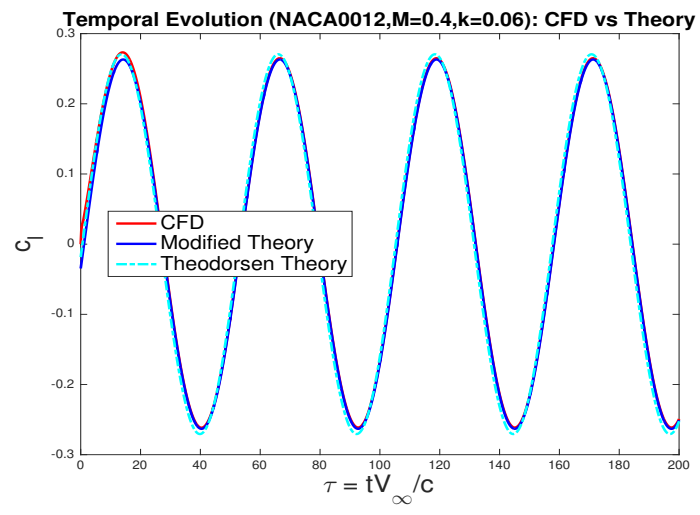

(c) $\mathrm{k}=0.06$

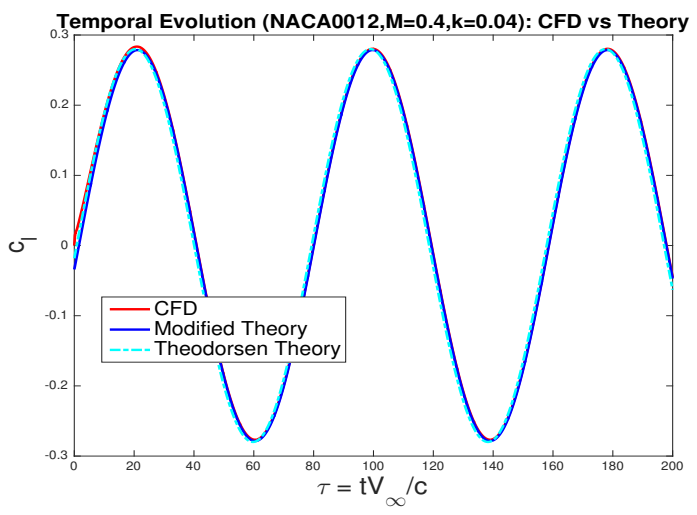

(b) $\mathrm{k}=0.04$

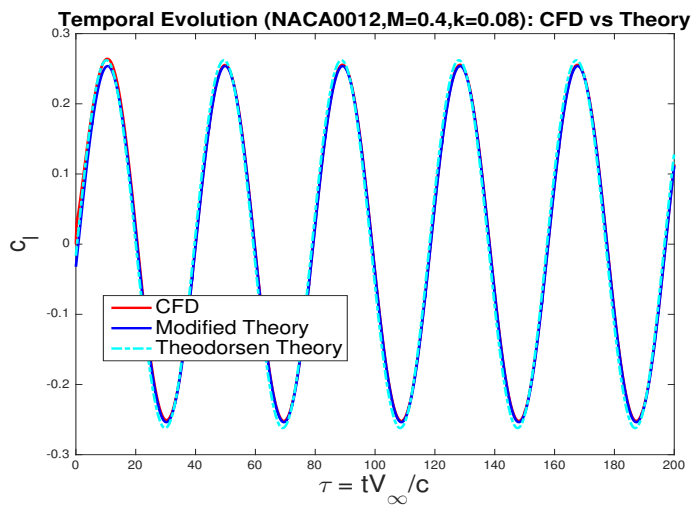

(d) $\mathrm{k}=0.08$

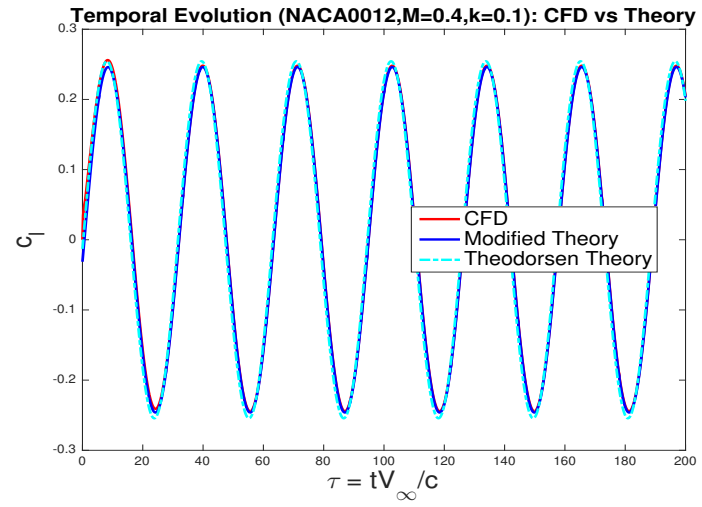

(e) $\mathrm{k}=0.1$

Figure 27: $C_{l}$ vs $\tau: \mathrm{M}=0.4$ 


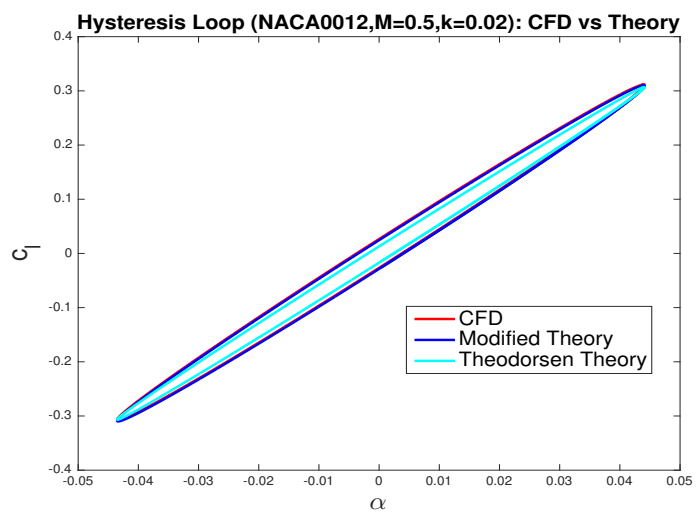

(a) $\mathrm{k}=0.02$

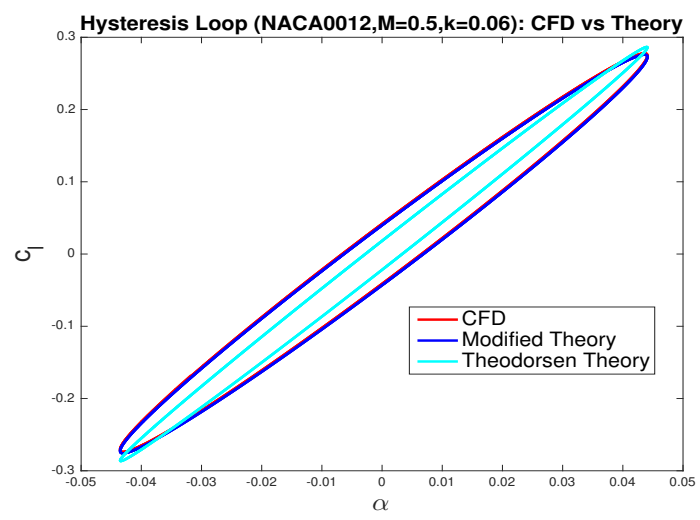

(c) $\mathrm{k}=0.06$

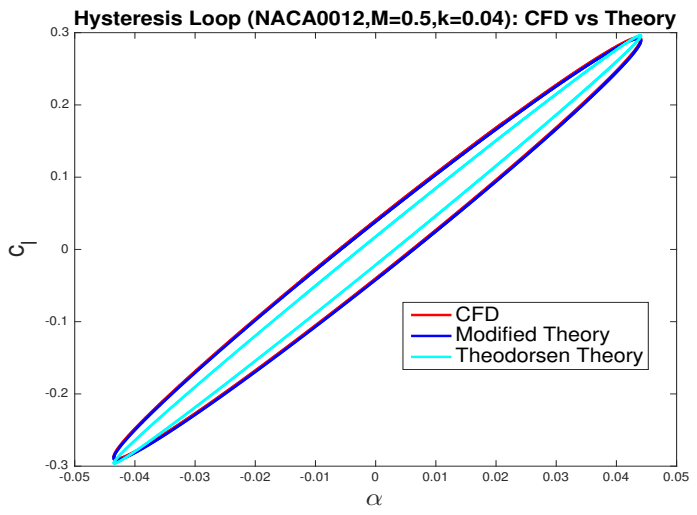

(b) $\mathrm{k}=0.04$

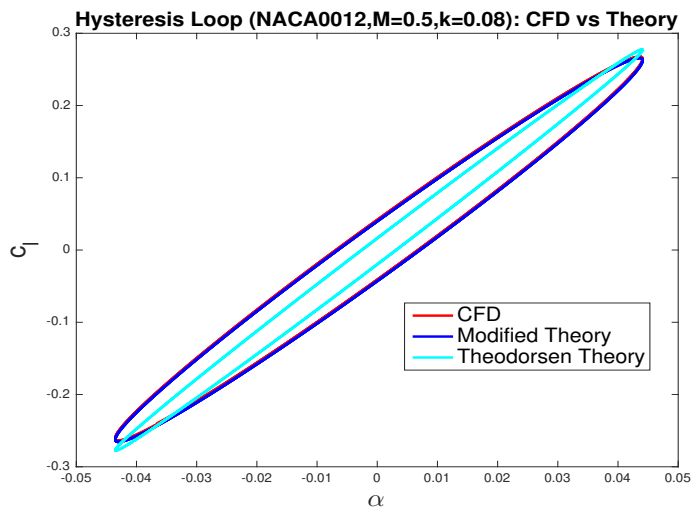

(d) $\mathrm{k}=0.08$

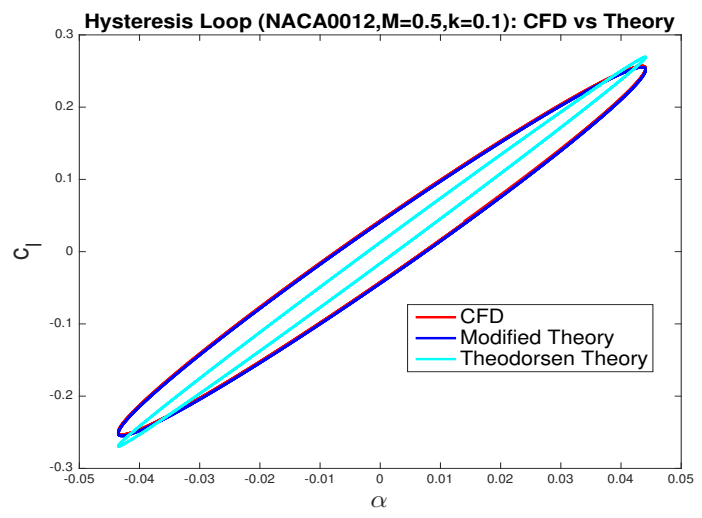

(e) $\mathrm{k}=0.1$

Figure 28: $C_{l}-\alpha$ hysteresis loop: $\mathrm{M}=0.5$ 


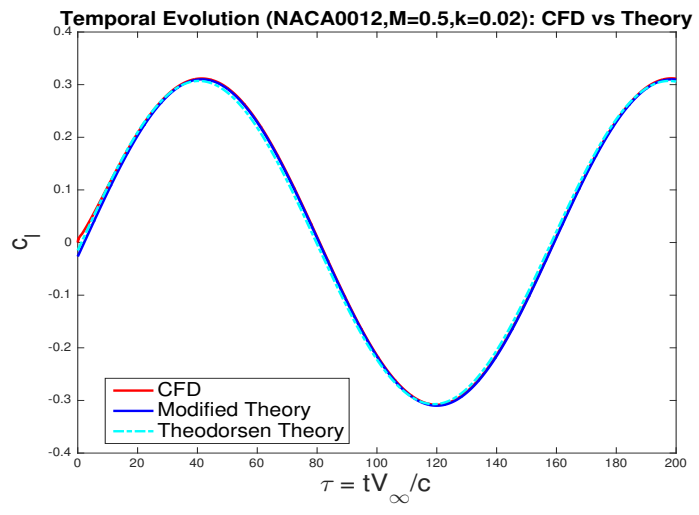

(a) $\mathrm{k}=0.02$

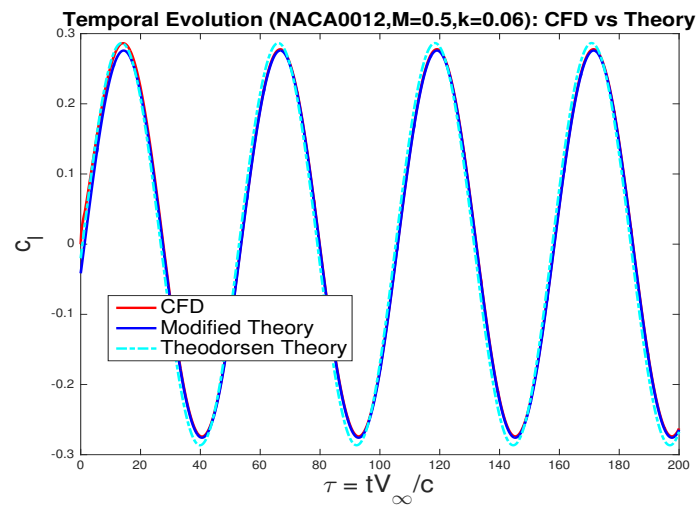

(c) $\mathrm{k}=0.06$

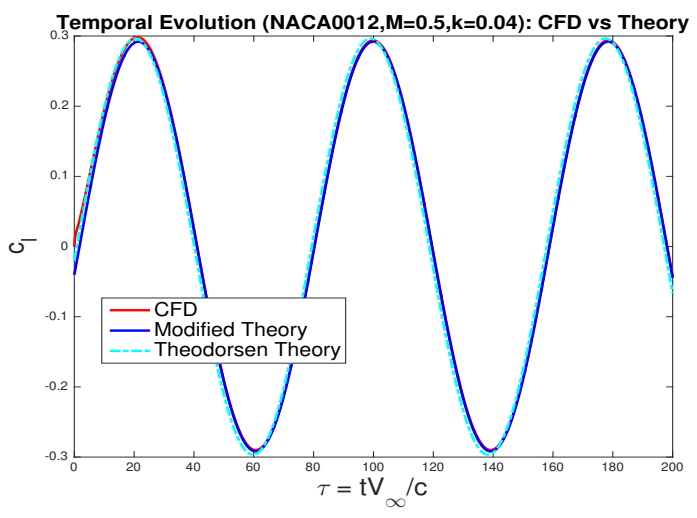

(b) $\mathrm{k}=0.04$

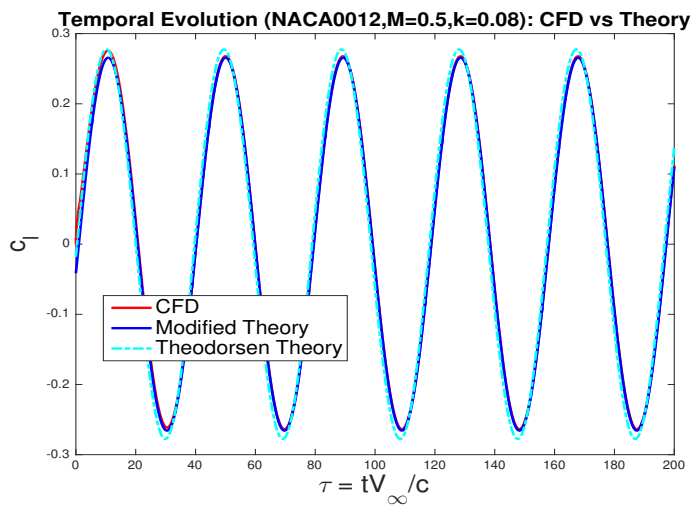

(d) $\mathrm{k}=0.08$

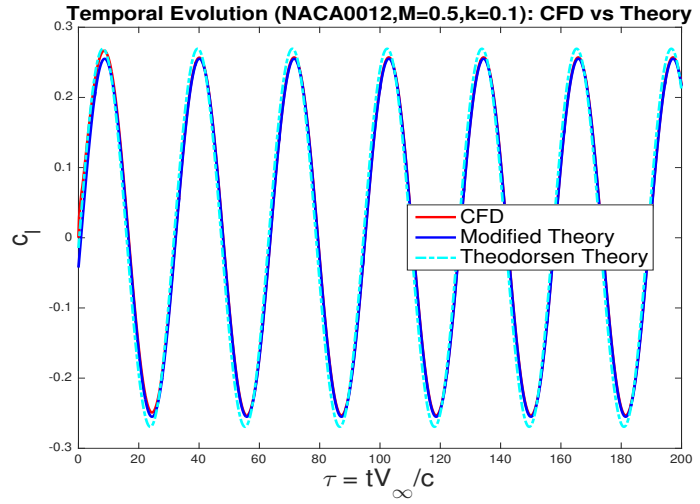

(e) $\mathrm{k}=0.1$

Figure 29: $C_{l}$ vs $\tau: \mathrm{M}=0.5$ 


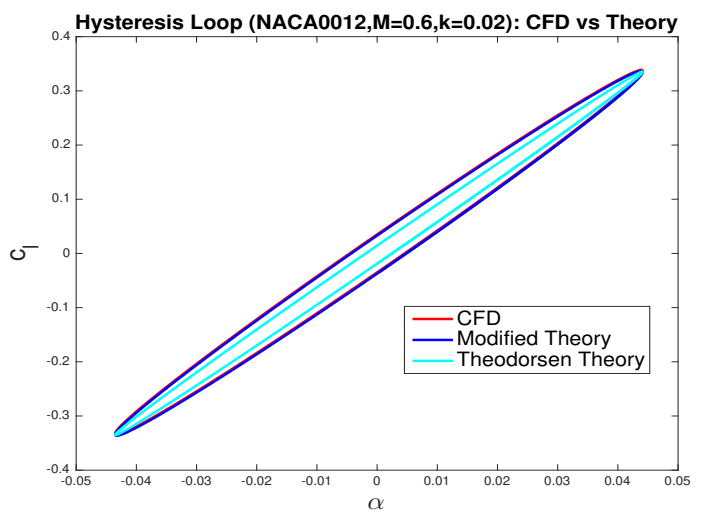

(a) $\mathrm{k}=0.02$

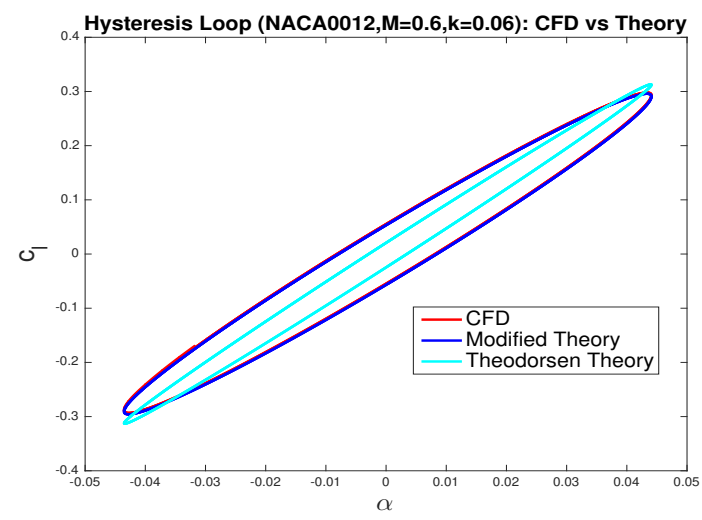

(c) $\mathrm{k}=0.06$

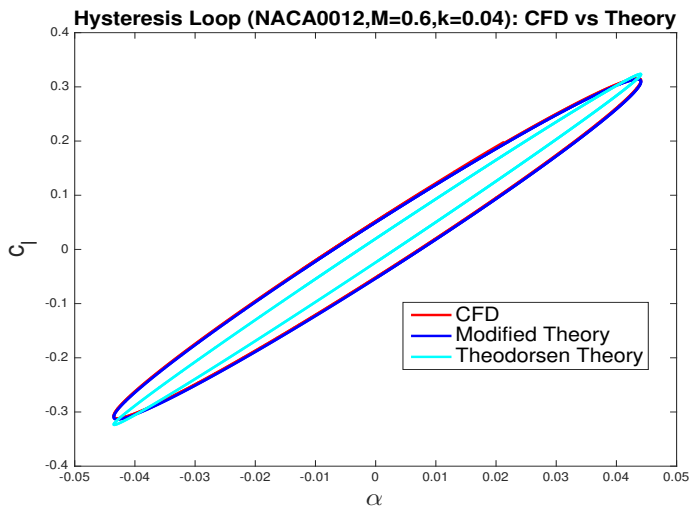

(b) $\mathrm{k}=0.04$

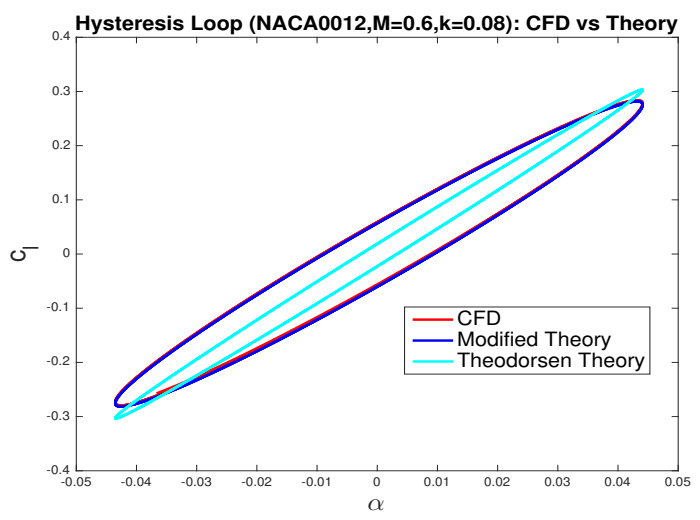

(d) $\mathrm{k}=0.08$

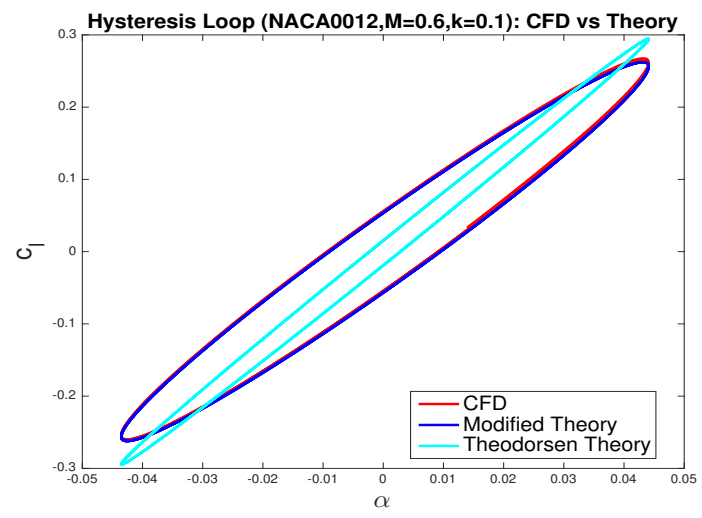

(e) $\mathrm{k}=0.1$

Figure 30: $C_{l}-\alpha$ hysteresis loop: $\mathrm{M}=0.6$ 


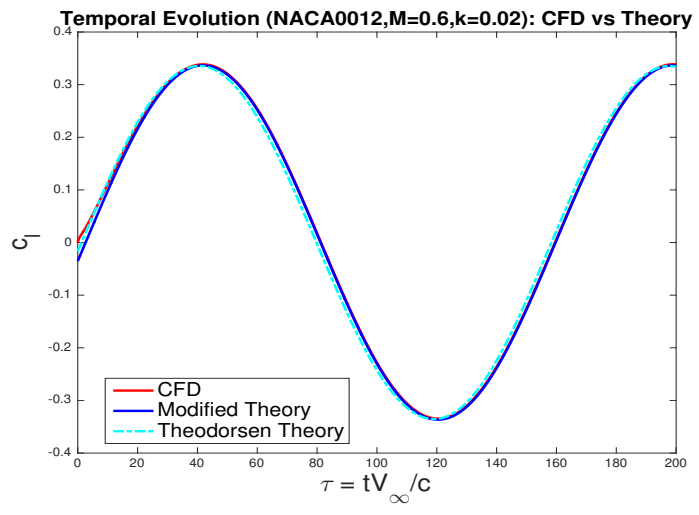

(a) $\mathrm{k}=0.02$

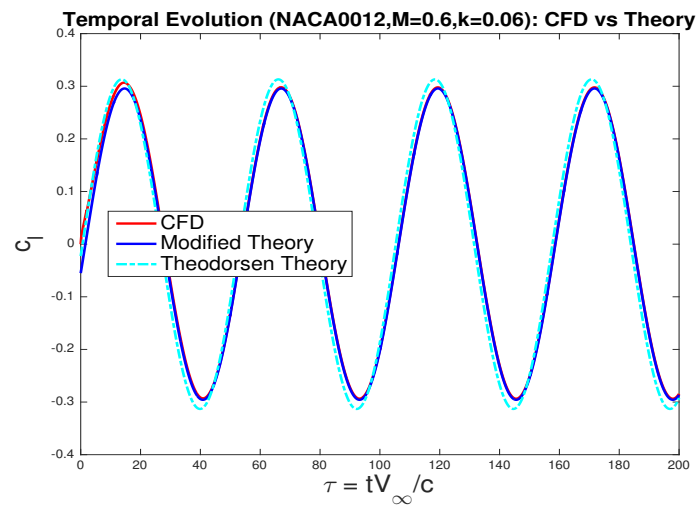

(c) $\mathrm{k}=0.06$

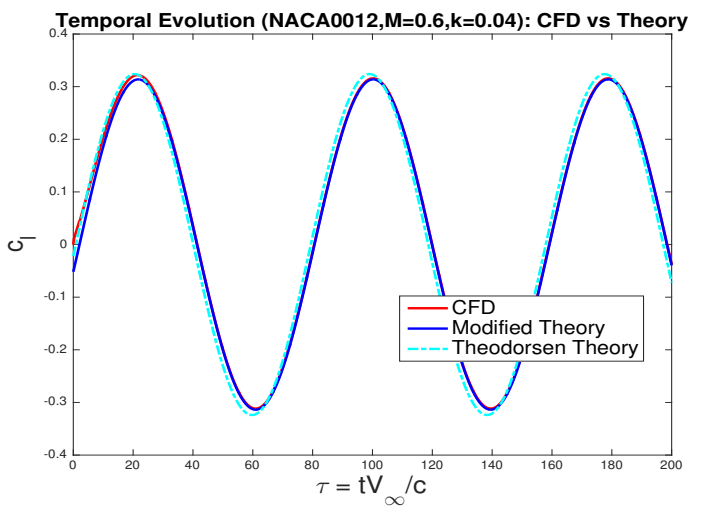

(b) $\mathrm{k}=0.04$

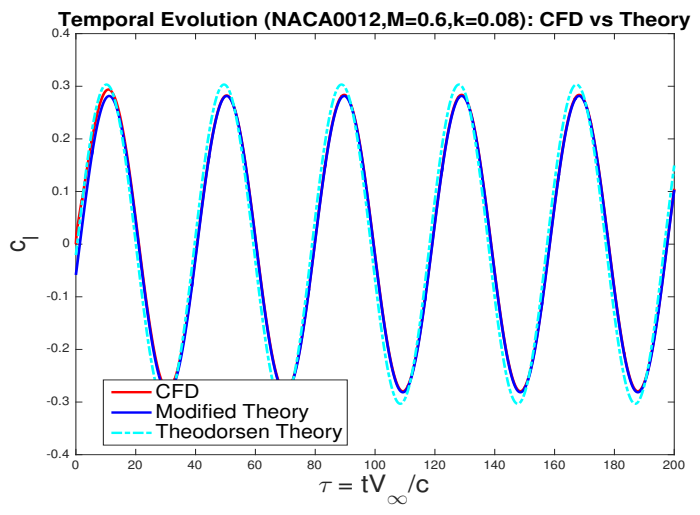

(d) $\mathrm{k}=0.08$

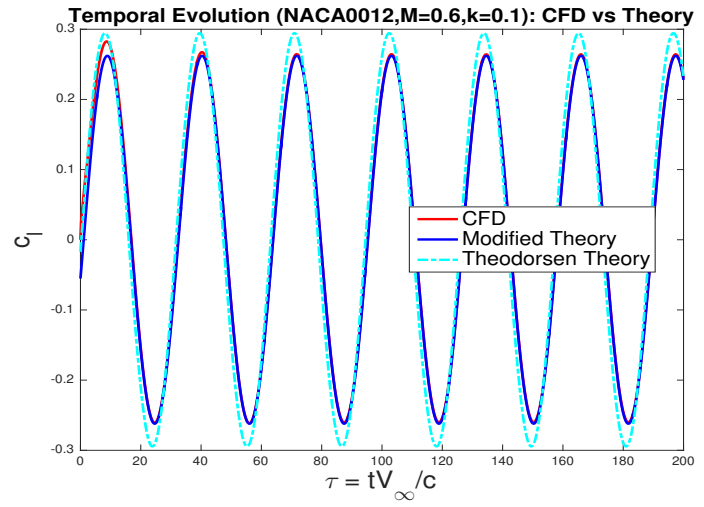

(e) $\mathrm{k}=0.1$

Figure 31: $C_{l}$ vs $\tau: \mathrm{M}=0.6$ 




(a) $\mathrm{k}=0.02$

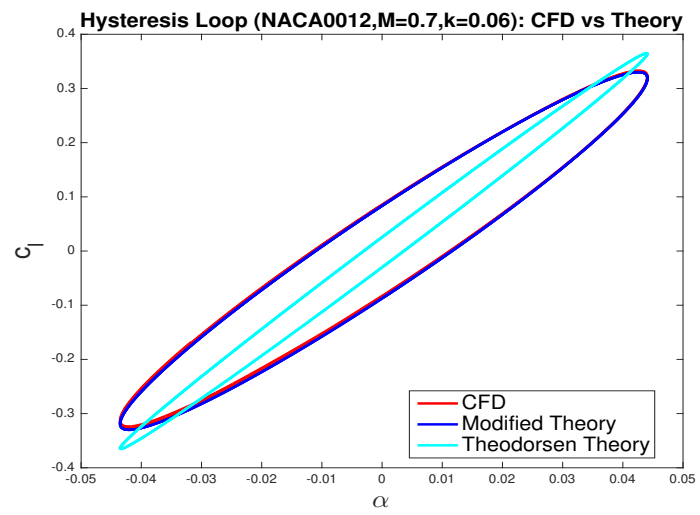

(c) $\mathrm{k}=0.06$

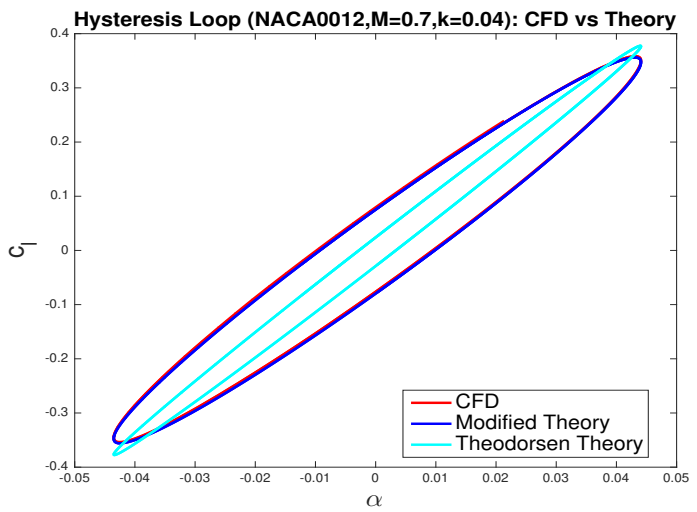

(b) $\mathrm{k}=0.04$



(d) $\mathrm{k}=0.08$

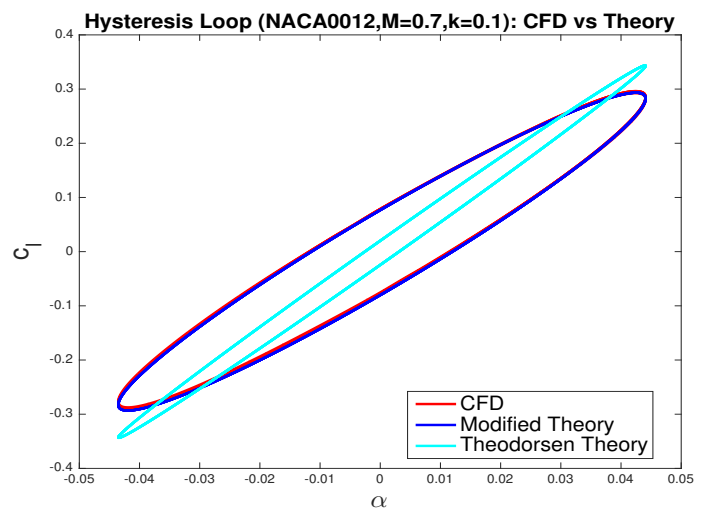

(e) $\mathrm{k}=0.1$

Figure 32: $C_{l}-\alpha$ hysteresis loop: $\mathrm{M}=0.7$ 


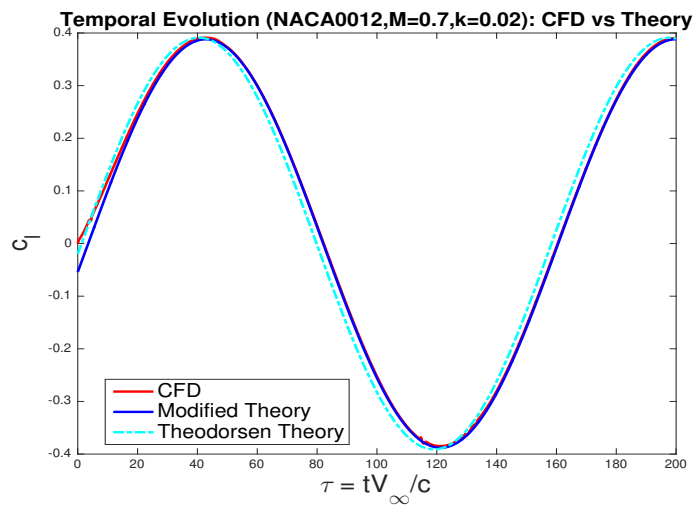

(a) $\mathrm{k}=0.02$

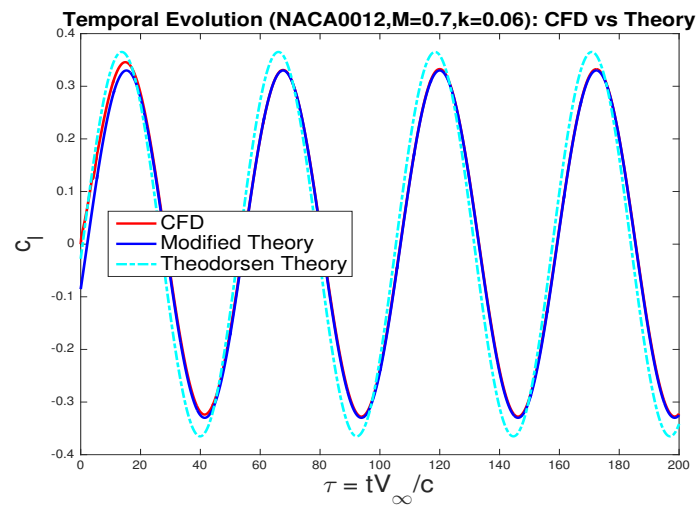

(c) $\mathrm{k}=0.06$

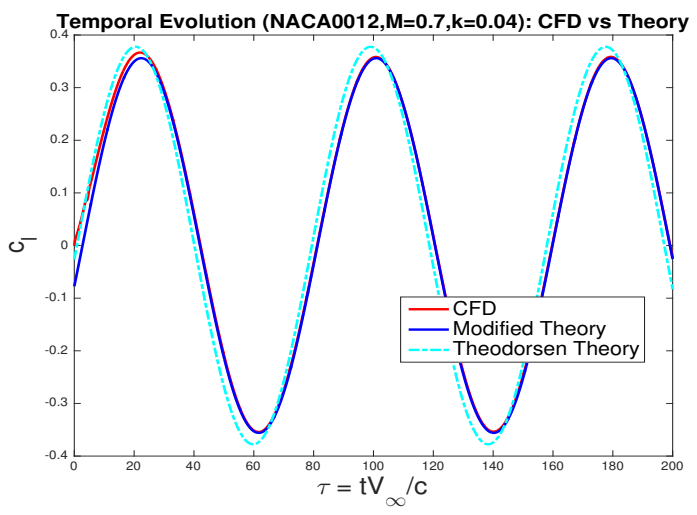

(b) $\mathrm{k}=0.04$

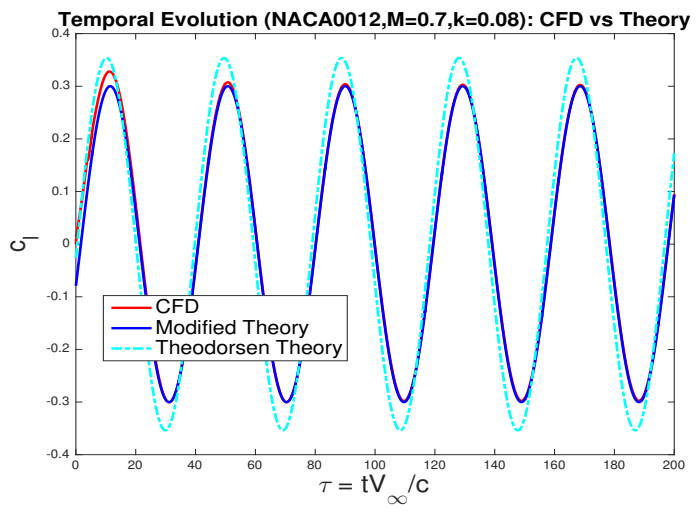

(d) $\mathrm{k}=0.08$

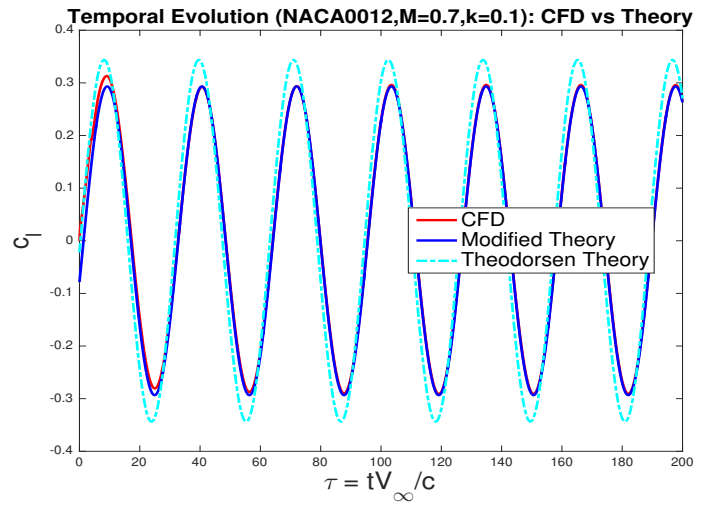

(e) $\mathrm{k}=0.1$

Figure 33: $C_{l}$ vs $\tau: \mathrm{M}=0.7$ 


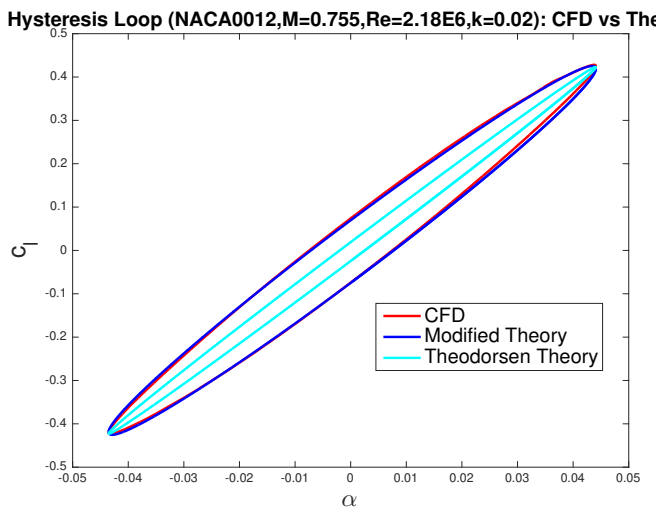

(a) $\mathrm{k}=0.02$

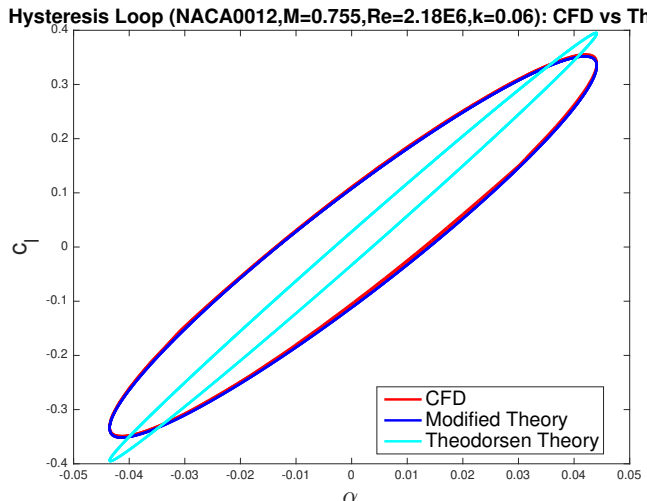

(c) $\mathrm{k}=0.06$

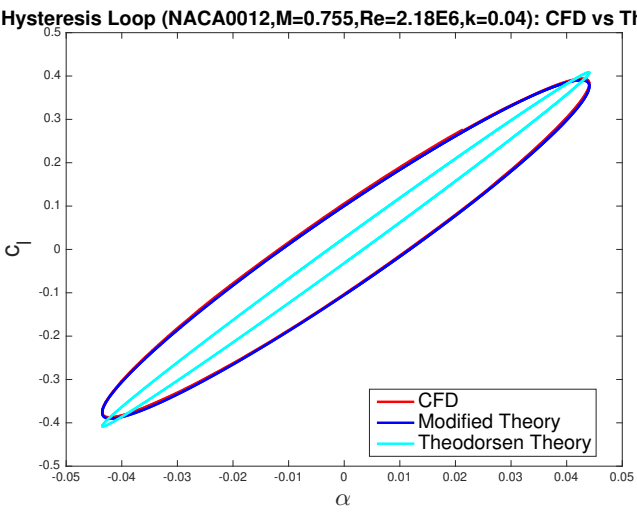

(b) $\mathrm{k}=0.04$



(d) $\mathrm{k}=0.08$



(e) $\mathrm{k}=0.1$

Figure 34: $C_{l}-\alpha$ hysteresis loop: $\mathrm{M}=0.755$ 


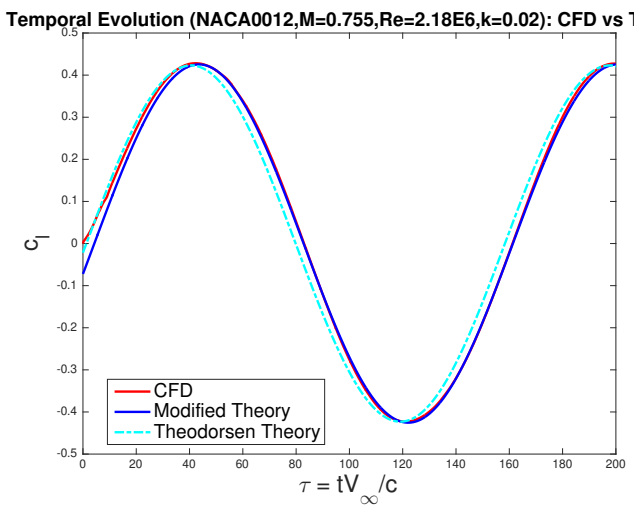

(a) $\mathrm{k}=0.02$

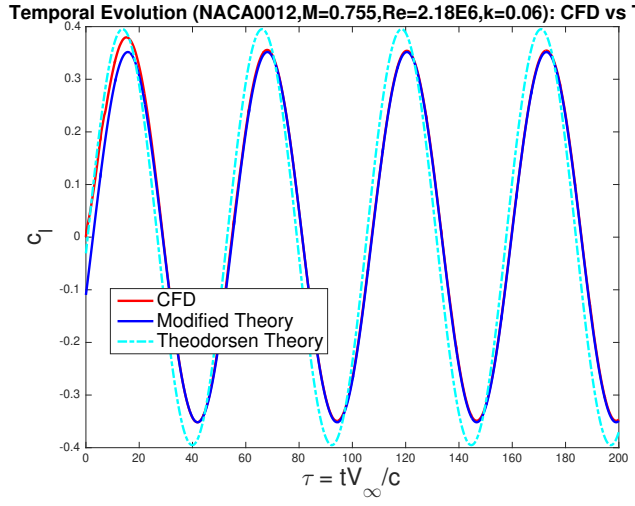

(c) $\mathrm{k}=0.06$

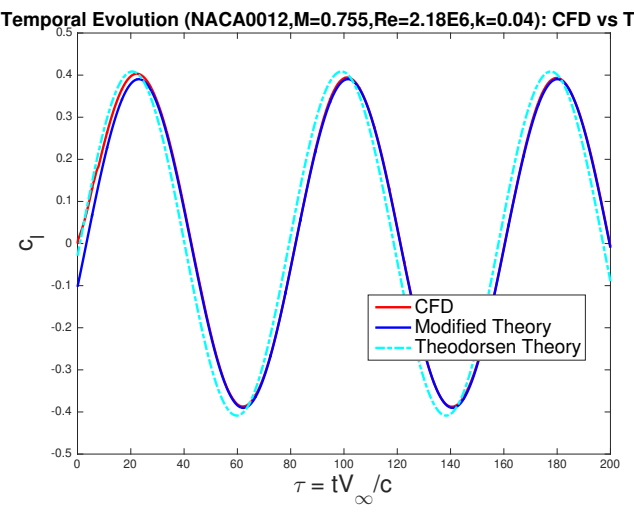

(b) $\mathrm{k}=0.04$

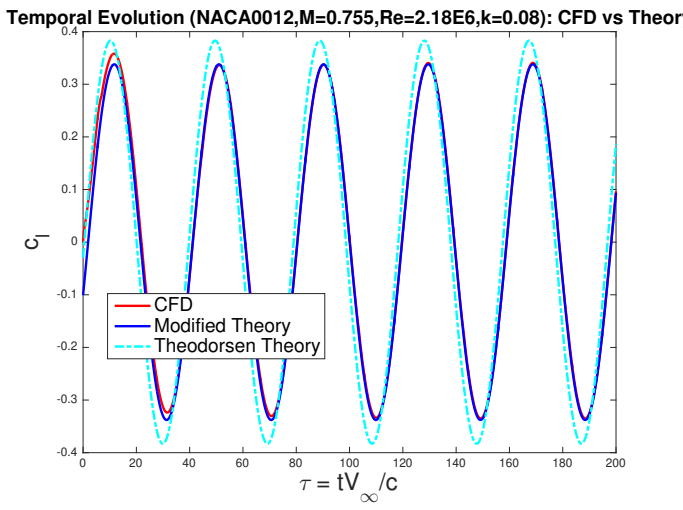

(d) $\mathrm{k}=0.08$

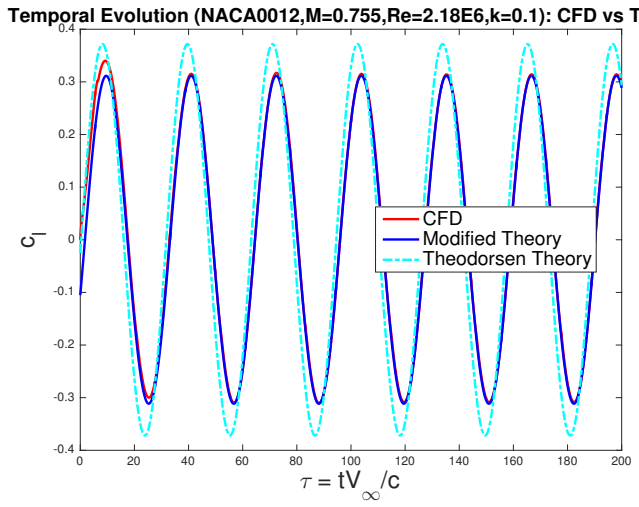

(e) $\mathrm{k}=0.1$

Figure 35: $C_{l}$ vs $\tau: \mathrm{M}=0.755$ 

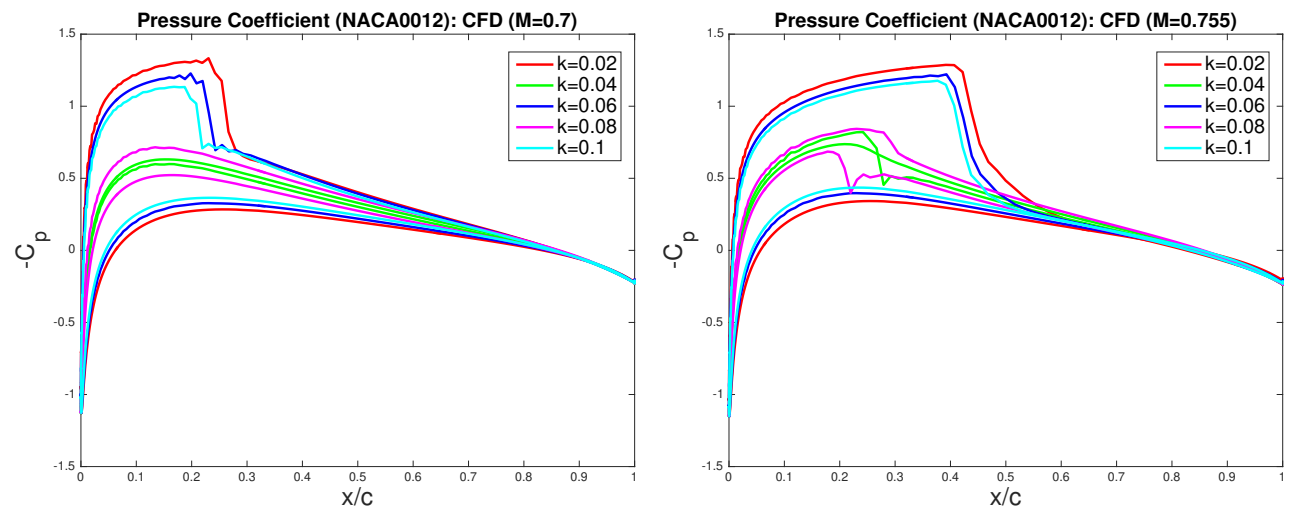

Figure 36: Pressure distribution over NACA0012: $C_{p}$

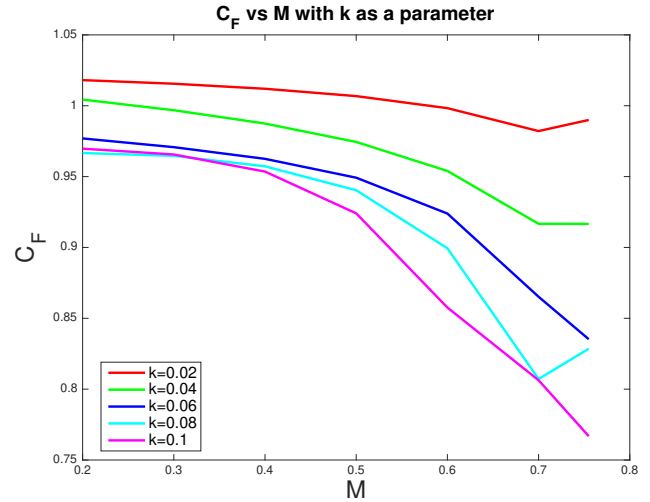

(a) $C_{F}$



(b) $C_{G}$

Figure 37: Variation of $C_{F}$ and $C_{G}$ with $\mathrm{M}$ with k as a parameter



(a) $C_{F}$



(b) $C_{G}$

Figure 38: Variation of $C_{F}$ and $C_{G}$ with k with $\mathrm{M}$ as a parameter 